\title{
Complexity of Manipulation, Bribery, and Campaign Management in Bucklin and Fallback Voting*
}

\author{
Piotr Faliszewski \\ AGH University \\ Krakow, Poland
}

\author{
Yannick Reisch, Jörg Rothe, and Lena Schend \\ Heinrich-Heine-Universität Düsseldorf \\ Düsseldorf, Germany
}

July 27, 2013

\begin{abstract}
A central theme in computational social choice is to study the extent to which voting systems computationally resist manipulative attacks seeking to influence the outcome of elections, such as manipulation (i.e., strategic voting), control, and bribery. Bucklin and fallback voting are among the voting systems with the broadest resistance (i.e., NP-hardness) to control attacks. However, only little is known about their behavior regarding manipulation and bribery attacks. We comprehensively investigate the computational resistance of Bucklin and fallback voting for many of the common manipulation and bribery scenarios; we also complement our discussion by considering several campaign management problems for Bucklin and fallback.
\end{abstract}

\section{Introduction}

A central theme in computational social choice (see, e.g., the bookchapter by Brandt et al. [BCE13]) is to study the extent to which voting systems computationally resist manipulative attacks that seek to influence the outcome of elections, such as manipulation (i.e., strategic voting), control, and bribery. In manipulation (introduced by Bartholdi et al. [BTT89, BO91] and, more generally, by Conitzer et al. [CSL07]; see, e.g., the survey by Faliszewski and Procaccia [FP10]), voters try to do so by casting insincere votes. In control (introduced by Bartholdi et al. [BTT92], see also Hemaspaandra et al. [HHR07]), an election chair tries to influence the election outcome by changing the structure of the election via adding/deleting/partitioning either candidates or voters. In bribery (introduced by Faliszewski [FHH09]), an external agent tries to influence the election outcome by bribing certain voters without exceeding some given budget. Since these types of influence are often possible in principle for many voting systems, it has been studied to what extent computational hardness can provide some kind of protection.

Bucklin and fallback voting [BS09] are among the voting systems with the broadest resistance (i.e., NP-hardness_1 ) to control attacks (see the work of Erdélyi et al. [ER10, EPR11, EF10b,

\footnotetext{
${ }^{*}$ This work was supported in part by DFG grant RO 1202/15-1, by a DAAD grant for a PPP project in the PROCOPE program, by NCN grants 2012/06/M/ST1/00358, 2011/03/B/ST6/01393, and by the AGH University grant 11.11.230.015.

${ }^{1}$ Resistance to manipulative actions is most often meant to be NP-hardness in the literature. Being a worst-case
} 
EFRS12]). ${ }_{-}^{2}$ However, only little is known about the behavior of these two voting systems regarding manipulation and bribery attacks; Schlotter et al. [SFE11] have studied them with respect to campaign management, focusing on shift bribery and support bribery. We comprehensively investigate the computational resistance of Bucklin and fallback voting for many of the common manipulation and bribery scenarios. We also complement the results of Schlotter et al. [SFE11] by studying two other campaign-management problems, namely swap bribery and extension bribery.

\section{Preliminaries}

\subsection{Bucklin and Fallback Elections}

An election is a pair $(C, V)$, where $C=\left\{c_{1}, \ldots, c_{m}\right\}$ is a set of $m$ candidates and $V=\left(v_{1}, \ldots, v_{n}\right)$ is a list of votes (or ballots) specifying the $n$ voters' preferences over the candidates in $C$. How these preferences are represented depends on the voting system used. We allow voters to be weighted, i.e., a nonnegative integer weight $w_{i}$ is associated with each vote $v_{i}$. For example, a vote $v_{i}$ of a voter with weight $w_{i}=3$ is counted as if three voters with unit weight would have cast the same ballot. An unweighted election is the special case of a weighted election where each voter has unit weight.

A voting system is a rule for how to determine the winner(s) of a given election. Here we focus on Bucklin and fallback voting only. Both systems use the notion of (weighted) majority threshold in $V$, which is defined by $\operatorname{maj}(V)=\lfloor W / 2\rfloor+1$, where $W=\sum_{i=1}^{n} w_{i}$ is the total weight of the votes in $V$. In Bucklin voting, votes are linear rankings of all candidates, denoted by, e.g., $c_{2}>c_{3}>c_{1}$, which means that this voter (strictly) prefers $c_{2}$ to $c_{3}$ and $c_{3}$ to $c_{1}$. We call the top position in a vote level 1, the next position level 2, and so on. Starting with the top position and proceeding level by level through the votes in $V$, we determine the smallest level $\ell$ such that some candidate(s) occur(s) in at least $\operatorname{maj}(V)$ votes up to this level. ${ }_{-}^{3}$ A bit more formally, for each candidate $c \in C$, the Bucklin score of $c$ in $(C, V)$, denoted by $\operatorname{score}_{(C, V)}^{i}(c)$, is the smallest level $k$ such that $c$ occurs in at least $\operatorname{maj}(V)$ votes within the first $k$ levels. Among the candidates from $C$ with smallest Bucklin score, say $\ell$, those occurring most often up to level $\ell$ are the Bucklin winners. If a candidate $c$ becomes a Bucklin winner on level $\ell$, we sometimes specifically call $c$ a level $\ell$ Bucklin winner.

Fallback voting is a hybrid voting systems designed by Brams and Sanver [BS09] to combine Bucklin with approval voting. Let us first define approval voting, which was proposed by Brams and Fishburn [BF78] (see also, e.g., [BF83, $\left.\mathrm{BEH}^{+} 10\right]$ for more background). In approval voting, votes in an election $(C, V)$ are approval vectors from $\{0,1\}^{\|C\|}$ indicating for each candidate $c \in C$ whether $c$ is approved ("1") by this voter or not ("0"). Every candidate with the highest approval score is an approval winner. For each vote $v \in V$, let $S_{v}$ denote the approval strategy of $v$, i.e., $S_{v} \subseteq C$ contains the candidates approved by $v$. In fallback voting, voters first approve or disapprove

measure only, NP-hardness does have its limitations. There are also a number of other approaches that challenge such NP-hardness results, surveyed in [RS]; for example, there are some experimental results on the control complexity of Bucklin and fallback voting [RS12].

${ }^{2}$ Other voting systems whose control complexity has thoroughly been studied include plurality, Condorcet, and approval voting [BTT92, HHR07], Llull and Copeland voting [FHHR09], a variant of approval voting known as SP-AV [ENR09], and normalized range voting [Men11].

${ }^{3}$ In simplified Bucklin voting, all these candidates win. However, we consider Bucklin voting in the unsimplified version where winners are determined by a slightly more involved procedure. Note that every Bucklin winner, as defined in the main text, also wins in simplified Bucklin voting, but not necessarily the other way round. 
of all candidates and then they provide a linear ranking of all approved candidates. For example, some voter might disapprove of $c_{1}$ and $c_{4}$, but approve of $c_{2}$ and $c_{3}$, preferring $c_{2}$ to $c_{3}$; this vote is denoted by $c_{2}>c_{3} \mid\left\{c_{1}, c_{4}\right\}$. To determine the winners in fallback voting, we first try to find the Bucklin winners when they exist. If so, all Bucklin winners are fallback winners. However, due to disapprovals it might happen that there is no Bucklin winner, and in that case all approval winners are fallback winners. A bit more formally, given a fallback election $(C, V)$, let $A(c)=\left\{v \in V \mid c \in S_{v}\right\}$ denote the set of voters that approve of candidate $c \in C$, let $A^{j}(c)$ denote the set of voters that approve of candidate $c$ up to the $j$ th level, and define

$$
\operatorname{score}_{(C, V)}(c)=\sum_{v_{i} \in A(c)} w_{i} \text { and } \operatorname{score}_{(C, V)}^{j}(c)=\sum_{v_{i} \in A^{j}(c)} w_{i} .
$$

The fallback score of $c$ in $(C, V)$ is the smallest level $k$ such that $\operatorname{score}_{(C, V)}^{k}(c) \geq \operatorname{maj}(V)$. Among the candidates from $C$ with smallest fallback score, say $\ell$, those occurring most often up to level $\ell$ are the (level $\ell$ ) fallback winners. Otherwise (i.e., if no candidate in $C$ satisfies $\operatorname{score}_{(C, V)}^{k}(c) \geq \operatorname{maj}(V)$ for any $k \leq m)$, all candidates $c$ with maximum $\operatorname{score}_{(C, V)}(c)$ are the fallback winners.

It is clear from the definition above that Bucklin elections are special fallback elections where all voters approve of all candidates. In other scenarios modeling tampering with election results (e.g., in control scenarios where the chair changes the structure of the election without changing the voters' preferences), this implies that NP-hardness results for control problems in Bucklin elections can be directly transferred to the same control problems in the more general fallback elections. In manipulation and bribery scenarios, however, such a direct transformation is not possible because the preferences of certain voters are changed, and we will show our results separately for both voting systems.

\subsection{Basics from Complexity Theory}

We assume the reader is familiar with the basic notions from complexity theory such as the complexity classes $\mathrm{P}$ and NP, the polynomial-time many-one $\left(\leq_{m}^{p}\right)$ and Turing $\left(\leq_{T}^{p}\right)$ reducibility, and with hardness and completeness with respect to $\leq_{m}^{p}$. For more background on complexity theory, see, e.g., the textbooks [Pap95, Rot05].

\section{Manipulation in Bucklin and Fallback Voting}

\subsection{Definitions and Overview of Results}

Conitzer et al. [CSL07] introduced the following decision problem to model manipulation by a coalition of weighted voters. For a given election system $\mathscr{E}$, define:

\begin{tabular}{ll}
\hline \multicolumn{2}{c}{ E-ConstruCtive CoAlitional Weighted Manipulation $(\mathscr{E}$-CCWM) } \\
\hline Given: $\quad \begin{array}{l}\text { A set } C \text { ondidates, a list } V \text { of nonmanipulative votes over } C \text { each having a } \\
\text { nonnegative integer weight, where } W_{V} \text { is the list of these weights, a list } W_{S} \text { of } \\
\text { the weights of } k \text { manipulators in } S \text { (whose votes over } C \text { are still unspecified) with } \\
\\
\quad V \cap S=\emptyset, \text { and a designated candidate } c \in C .\end{array}$ \\
Question: $\quad$ Can the votes in $S$ be set such that $c$ is the unique $\mathscr{E}$ winner of $(C, V \cup S)$ ? \\
\hline
\end{tabular}


Table 1: Overview of results for manipulation in Bucklin and fallback voting

\begin{tabular}{|c|c|c|c|c|}
\hline & \multicolumn{2}{|c|}{ Bucklin voting } & \multicolumn{2}{|c|}{ fallback voting } \\
\hline & complexity & reference & complexity & reference \\
\hline $\mathscr{E}$-CCUM & $\mathrm{P}$ & Thm. 3.4 & $\mathrm{P}$ & Prop. 3.2 \\
\hline $\mathscr{E}$-DCUM & $\mathrm{P}$ & Cor. $\underline{3.5}$ & $\mathrm{P}$ & Prop. $\underline{3.2}$ \\
\hline $\mathscr{E}-\mathrm{CCWM}$ & NP-complete & Thm. 3.7 & $\mathrm{P}$ & Prop. 3.6 \\
\hline $\mathscr{E}$-DCWM & $\mathrm{P}$ & Thm. 3.9 & $\mathrm{P}$ & Prop. $\underline{3.6}$ \\
\hline
\end{tabular}

The unweighted case $\mathscr{E}$-CCUM is the special case of $\mathscr{E}$-CCWM where all voters and manipulators have unit weight. By changing the question to "... such that $c$ is not a unique winner in $(C, V \cup S)$ ?," we obtain the destructive variants, $\mathscr{E}$-DCWM and $\mathscr{E}$-DCUM. If there is only one manipulator, we denote the corresponding problems by $\mathscr{E}$-CUM, $\mathscr{E}$-CWM, $\mathscr{E}$-DUM, and $\mathscr{E}$-DWM; these problems were first studied by Bartholdi et al. [BTT89, BO91].

The following proposition follows immediately from the definitions.

Proposition 3.1 1. $\mathscr{E}-C U M \leq_{m}^{p} \mathscr{E}-C C U M \leq_{m}^{p} \mathscr{E}-C C W M$.

2. $\mathscr{E}-D U M \leq_{m}^{p} \mathscr{E}-D C U M \leq_{m}^{p} \mathscr{E}-D C W M$.

3. $\mathscr{E}-D U M \leq_{T}^{p} \mathscr{E}-C U M, \mathscr{E}-D W M \leq_{T}^{p} \mathscr{E}-C W M$.

4. $\mathscr{E}-D C U M \leq_{T}^{p} \mathscr{E}-C C U M$.

5. $\mathscr{E}-D C W M \leq_{T}^{p} \mathscr{E}-C C W M$.

Table 1 gives an overview of our results for manipulation in Bucklin and fallback voting.

\subsection{Results for Unweighted Manipulation}

In fallback elections, manipulators that try to make a certain candidate the winner by changing their votes can follow a simple strategy: They can limit their approval strategy to only this candidate and thus preclude all other candidates from gaining points from their votes. It is easy to see that if this attempt is not successful, no other way of constructing the manipulators' votes can make their designated candidate win. This means that fallback-CCUM is in P, which implies P-membership for fallback-CUM, fallback-DCUM, and fallback-DUM as well (with Proposition $\underline{3.1}$ ). We state this observation in the following proposition.

Proposition 3.2 Fallback-CCUM, fallback-CUM, fallback-DCUM, and fallback-DUM are in P.

In Bucklin elections, however, the argumentation is more involved, since the manipulators do not have the possibility to preclude any candidate from gaining points from their votes. So the manipulators' votes have to be carefully constructed to ensure that no other candidate than the designated candidate gains too much points on the relevant levels.

Nevertheless, we can show that Bucklin-CCUM is in $\mathrm{P}$ by adapting an algorithm for simplifiedBucklin-CCUM that is due to Xia et al. $\left[\mathrm{XZP}^{+} 09\right]$, see Algorithm $\underline{1}$. 


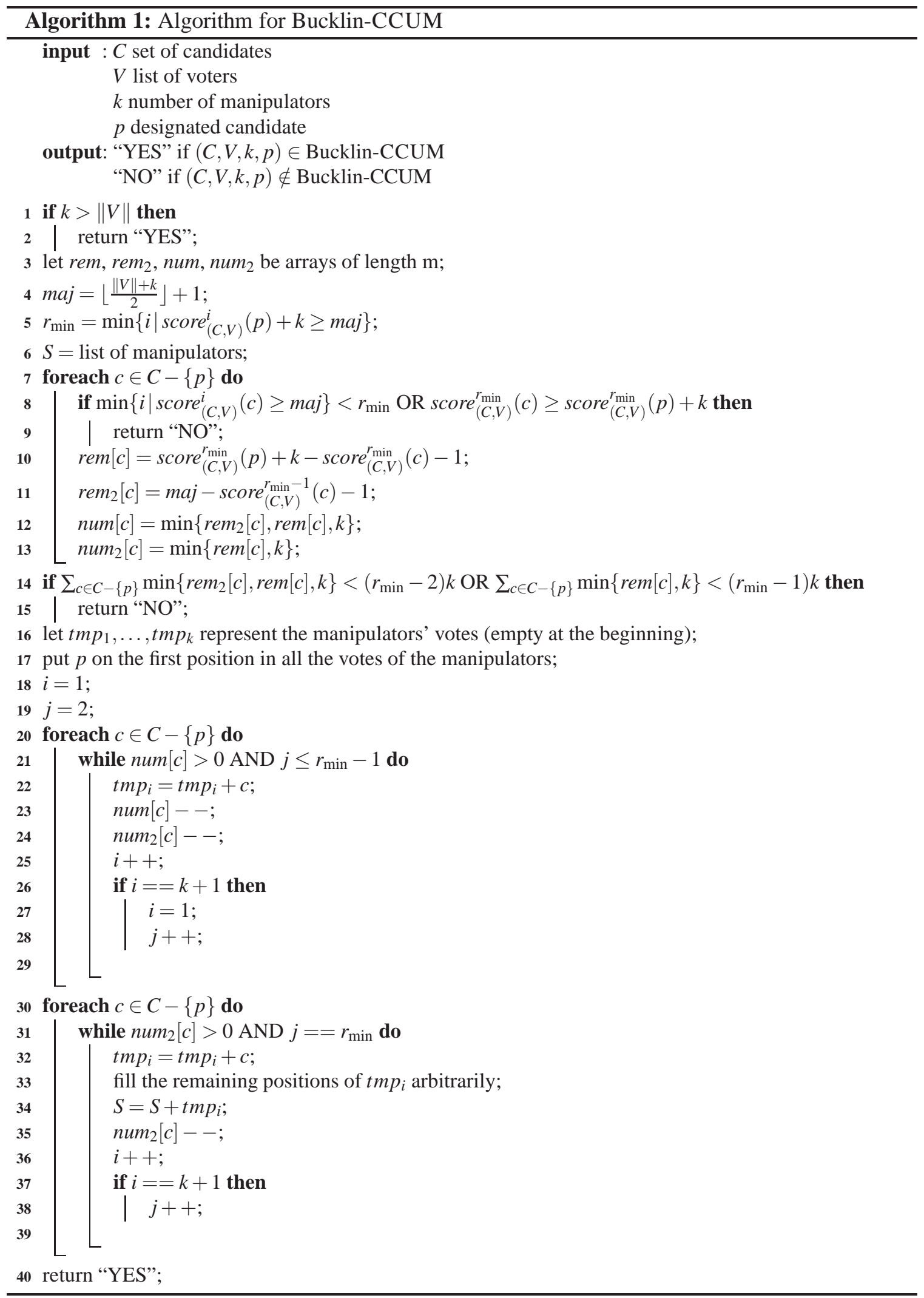


Before we prove that the presented algorithm is correct and in $\mathrm{P}$, we show the following useful lemma.

Lemma 3.3 Considering the notation $C, V, k, p$, rem, rem 2 , num, num $2, r_{\min }, S$, and maj as in Algorithm $\underline{1}$, it holds that:

1. If $k>\|V\|$ then $(C, V, k, p) \in$ Bucklin-CCUM.

2. If there is a candidate $c \in C-\{p\}$ with

(a) $\min \left\{i \mid \operatorname{score}_{(C, V)}^{i}(c) \geq \operatorname{maj}\right\}<r_{\min }$ or

(b) $\operatorname{score}_{(C, V)}^{r_{\min }}(c) \geq \operatorname{score}_{(C, V)}^{r_{\min }}(p)+k$,

then $(C, V, k, p) \notin$ Bucklin-CCUM.

3. $(C, V, k, p) \notin B u c k l i n-C C U M$ if and only if

(a) $\sum_{c \in C-\{p\}} \min \left\{\operatorname{rem}[c], \operatorname{rem}_{2}[c], k\right\}<\left(r_{\min }-2\right) k$ or

(b) $\sum_{c \in C-\{p\}} \min \{\operatorname{rem}[c], k\}<\left(r_{\min }-1\right) k$.

Proof. Note that $r_{\text {min }}$ denotes the smallest level on which candidate $p$ reaches the majority threshold maj in the manipulated election assuming that all manipulators position $p$ on the first place. So $r_{\min }$ is the smallest level on which $p$ can win. This implies that $\operatorname{score}_{(C, V)}^{r_{\min }}(p)+k$ is the number of points $p$ has to win the election with. Now we can show the three claims.

1. If the number of manipulators is bigger than the number of truthful voters, a successful manipulation is always possible. The manipulators simply position $p$ on the first place in their vote and $p$ reaches the majority threshold already on the first level. So $(C, V, k, p) \in$ BucklinCCUM trivially holds.

2. Let $c \in C-\{p\}$ be an arbitrary candidate.

(a) It holds that $\min \left\{i \mid \operatorname{score}_{(C, V)}^{i}(c) \geq \operatorname{maj}\right\}<r_{\min }$ : That means that we have a candidate $c$ that reaches maj votes on an earlier level than $p$ and $c$ does so even without the manipulators' votes. Thus $(C, V, k, p) \notin$ Bucklin-CCUM.

(b) It holds that $\operatorname{score}_{(C, V)}^{r_{\min }}(c) \geq \operatorname{score}_{(C, V)}^{r_{\min }}(p)+k$ : This means that $c$ gets at least as many points from the truthful voters on the exact level $p$ would have to win the manipulated election as $p$ gains in the election where the manipulators' votes have already been added. That means that $p$ cannot be made the unique winner of the manipulated election and thus $(C, V, k, p) \notin$ Bucklin-CCUM holds.

3. The array rem indicates for every candidate $c$ how many further points $c$ can gain without exceeding $\operatorname{score}_{(C, V)}^{r_{\min }}(p)$ on level $r_{\min }$. The array $\operatorname{rem}_{2}$, on the other hand, indicates for every candidate $c$ how many further points $c$ may gain without exceeding maj on the levels 1 to $\left(r_{\min }-1\right)$. For all candidates, rem and $\mathrm{rem}_{2}$ contain positive numbers. Since every candidate can gain $k$ points from the manipulators' votes, $\operatorname{num}[c]=\min \left\{r e m[c], \operatorname{rem}_{2}[c], k\right\}$ is 
the number of manipulators that may have candidate $c$ in the first $\left(r_{\min }-1\right)$ positions of their votes without preventing $p$ from winning. Analogously, $\operatorname{num}_{2}[c]=\min \{\operatorname{rem}[c], k\}$ is the number of manipulators that can place $c$ among their top $r_{\min }$ positions without preventing $p$ from winning. We have that num $_{2}[c] \geq$ num $[c]$ for all $c \in C-\{p\}$. We now show the equivalence.

From right to left:

(a) Suppose that $\sum_{c \in C-\{p\}} \min \left\{\operatorname{rem}[c], \operatorname{rem}_{2}[c], k\right\}<\left(r_{\min }-2\right) k$. In this case, it is not possible to fill the remaining $\left(r_{\min }-2\right) k$ positions (positions 2 to $\left.\left(r_{\min }-1\right)\right)$ in the manipulators' votes without having for at least one candidate $d \in C-\{p\}$ that either

$$
\begin{gathered}
\operatorname{rem}_{2}[d]-\operatorname{score}_{(C, S)}^{r_{\min }-1}(d)<0 \quad \text { or } \\
\operatorname{rem}[d]-\operatorname{score}_{(C, S)}^{r_{\min }}(d)<0
\end{gathered}
$$

holds. That is equivalent to either

$$
\begin{array}{r}
\operatorname{maj}-\operatorname{score}_{(C, V)}^{r_{\min }-1}(d)-1-\operatorname{score}_{(C, S)}^{r_{\min }-1}(d)<0 \\
\operatorname{score}_{(C, V)}^{r_{\min }}(p)+k-\operatorname{score}_{(C, V)}^{r_{\min }}(d)-1-\operatorname{score}_{(C, S)}^{r_{\min }}(d)<0,
\end{array}
$$

which in turn is equivalent to either

$$
\begin{gathered}
\operatorname{score}_{(C, V \cup S)}^{r_{\min }-1}(d)=\operatorname{score}_{(C, V)}^{r_{\min }-1}(d)+\operatorname{score}_{(C, S)}^{r_{\min }-1}(d)>m a j-1 \\
\operatorname{score}_{(C, V \cup S)}^{r_{\min }}(d)=\operatorname{score}_{(C, V)}^{r_{\min }}(d)+\operatorname{score}_{(C, S)}^{r_{\min }}(d)>\operatorname{score}_{(C, V)}^{r_{\min }}(p)+k-1 .
\end{gathered}
$$

So we have that either $d$ is a Bucklin winner in the manipulated election on a smaller level than $r_{\min }$, or it holds that on level $r_{\min }$ candidate $d$ might have at least as many points as $p$. Thus $(C, V, k, p) \notin$ Bucklin-CCUM.

(b) Suppose that $\sum_{c \in C-\{p\}} \min \{\operatorname{rem}[c], k\}<\left(r_{\min }-1\right) k$. In this case, it is not possible to fill the remaining $\left(r_{\min }-1\right) k$ positions (positions 2 to $\left.r_{\text {min }}\right)$ in the manipulators' votes without having for at least one candidate $d \in C-\{p\}$ :

$$
\begin{aligned}
& \operatorname{rem}[d]-\operatorname{score}_{(C, S)}^{r_{\min }}(d)<0 \\
\Leftrightarrow & \operatorname{score}_{(C, V)}^{r_{\min }}(p)+k-\operatorname{score}_{(C, V)}^{r_{\min }}(d)-1-\operatorname{score}_{(C, S)}^{r_{\min }}(d)<0 \\
\Leftrightarrow & \operatorname{score}_{(C, V \cup S)}^{r_{\text {min }}}(d)=\operatorname{score}_{(C, V)}^{r_{\min }}(d)+\operatorname{score}_{(C, S)}^{r_{\min }}(d)>\operatorname{score}_{(C, V)}^{r_{\text {min }}}(p)+k-1 .
\end{aligned}
$$

So we have that $d$ can have at least as many points as $p$ on level $r_{\min }$. So $(C, V, k, p) \notin$ Bucklin-CCUM.

From left to right: We show the contrapositive. Assume that both

(a) $\sum_{c \in C-\{p\}} \min \left\{\operatorname{rem}[c], \operatorname{rem}_{2}[c], k\right\} \geq\left(r_{\min }-2\right) k$ and

(b) $\sum_{c \in C-\{p\}} \min \{\operatorname{rem}[c], k\} \geq\left(r_{\min }-1\right) k$

hold. Then we can fill positions 2 to $r_{\min }$ of the manipulators' votes in a way that for all candidates $e \in C-\{p\}$ the following holds:

$$
\begin{aligned}
& \operatorname{rem}_{2}[e]-\operatorname{score}_{(C, S)}^{r_{\min }-1}(e) \geq 0 \quad \text { and } \\
& \operatorname{rem}[e]-\operatorname{score}_{(C, S)}^{r_{\min }}(e) \geq 0,
\end{aligned}
$$


which is equivalent to

$$
\begin{gathered}
\operatorname{score}_{(C, V \cup S)}^{r_{\min }-1}(e)=\operatorname{score}_{(C, V)}^{r_{\min }-1}(e)+\operatorname{score}_{(C, S)}^{r_{\min }-1}(e) \leq m a j-1 \\
\operatorname{score}_{(C, V \cup S)}^{r_{\min }}(e)=\operatorname{score}_{(C, V)}^{r_{\min }}(e)+\operatorname{score}_{(C, S)}^{r_{\min }}(e) \leq \operatorname{score}_{(C, V)}^{r_{\min }}(p)+k-1 .
\end{gathered}
$$

So we have that $(C, V, k, p) \in$ Bucklin-CCUM.

This completes the proof.

Now we are ready to show that Algorithm 1 is in P and correctly solves Bucklin-CCUM.

Theorem 3.4 Algorithm 1 has a runtime of $\mathscr{O}\left(m^{2}+n m\right)$ and decides Bucklin-CCUM, and thus this problem is in $\mathrm{P}$.

Proof. It follows immediately from Lemma $\underline{3.3}$ that Algorithm $\underline{1}$ is correct. It is also clear that it always terminates. To compute the needed scores $\operatorname{score}_{(C, V)}^{i}(c)$ for all candidates $c$ and every level $i$, $\mathscr{O}\left(m^{2}+n m\right)$ steps are needed. The for-loop in line 7 needs $\mathscr{O}(m)$ steps, while the other two for-loops in lines 20 and 30 need $\mathscr{O}(\mathrm{km})$ steps. Since the loops are only run through when $k \leq n$, we have a runtime of $\mathscr{O}(\mathrm{nm})$ for the loops, which implies that the algorithm has a runtime of $\mathscr{O}\left(m^{2}+n m\right)$ in total.

With Theorem $\underline{3.4}$ and Proposition $\underline{3.1}$ we have the following corollary.

Corollary 3.5 Bucklin-CUM, Bucklin-DUM, Bucklin-CCUM, and Bucklin-DCUM are in P.

\subsection{Results for Weighted Manipulation}

In this section we analyze the complexity of weighted manipulation in Bucklin and fallback voting.

With the same argumentation as that given at the beginning of Section 3.2, it is easy to see that in fallback elections the weighted manipulation problems can be decided efficiently, namely in deterministic polynomial time: In the constructive, coalitional, weighted case, all the manipulators need to do is to approve of the designated candidate - if this attempt does not lead to the desired result, no other way of changing the manipulators' votes will. Again, with Proposition $\underline{3.1}$, the result for this case directly transfers to the constructive, weighted case with only one manipulator, and from these two constructive cases to the corresponding destructive cases. We state this observation in the following proposition.

Proposition 3.6 Fallback-CCWM, fallback-CWM, fallback-DCWM, and fallback-DWM are in $\mathrm{P}$.

In weighted Bucklin elections, on the other hand, a coalition of manipulators trying to make a certain candidate win is faced with a harder challenge, as the following result shows.

Theorem 3.7 Bucklin-CCWM is NP-complete. 
Proof. It is easy to see that Bucklin-CCWM is in NP. We show NP-hardness of this problem by a reduction from the problem PARTITION: Given a set $A=\{1, \ldots, k\}$ and a sequence $\left(a_{1}, \ldots, a_{k}\right)$ of nonnegative integers with $\sum_{i=1}^{k} a_{i}=2 K$ for a positive integer $K$, is there a set $A^{\prime} \subseteq A$ such that $\sum_{i \in A^{\prime}} a_{i}=\sum_{i \notin A^{\prime}} a_{i}=K$ ? PARTITION is well-known to be NP-complete (see, e.g., [GJ79]).

Let an instance of PARTITION be given by $A=\{1,2, \ldots, k\}$ and $\left(a_{1}, \ldots, a_{k}\right)$ with $\sum_{i=1}^{k} a_{i}=2 K$. Without loss of generality, we may assume that $a_{i} \geq 2$ for each $i \in A$. We construct the following instance of Bucklin-CCWM. The candidate set is $C=\{b, c, d, p\}$ and $p$ is the designated candidate. In $V$ we have three voters of the following form with a total weight of $6 K-2$ :

1. $c>p>d>b$ with weight $2 K$,

2. $c>d>p>b$ with weight $K-1$,

3. $b>d>p>c$ with weight $3 K-1$,

so the majority threshold in $(C, V)$ is reached with $\lfloor(6 K-2) / 2\rfloor+1=3 K$. For the first two levels, the scores of the candidates are given in Table $\underline{2}$, and the unique level 2 Bucklin winner in $(C, V)$ is $d$. Furthermore, there are $k$ manipulators in $S$ with weights $a_{1}, a_{2}, \ldots, a_{k}$, which are given in our Bucklin-CCWM instance.

Table 2: Level $i$ scores in $(C, V)$ for $i \in\{1,2\}$ and the candidates in $C$

\begin{tabular}{lccccc}
\hline & $b$ & $c$ & $d$ & $p$ \\
score $^{1}$ & $3 K-1$ & & $3 K-1$ & 0 & 0 \\
score $^{2}$ & $3 K-1$ & $3 K-1$ & $4 K-2$ & $2 K$ \\
\hline
\end{tabular}

We claim that $\left(A,\left(a_{1}, a_{2}, \ldots, a_{k}\right)\right) \in$ PARTITION if and only if $p$ can be made the unique Bucklin winner in $(C, V \cup S)$.

From left to right: Assume that there is a subset $A^{\prime} \subseteq A$ with $\sum_{i \in A^{\prime}} a_{i}=K$. The majority threshold in $(C, V \cup S)$ is $\lfloor(6 K-2+2 K) / 2\rfloor+1=4 K$. Let the votes of the manipulators be of the following form

- $p>c>b>d$ for all manipulators with weight $a_{i}$ for $i \in A^{\prime}$, and

- $p>b>c>d$ for the remaining manipulators.

For the first two levels, the scores of the candidates in $(C, V \cup S)$ are given in Table $\underline{3}$. It follows that $p$ is the unique level 2 Bucklin winner in $(C, V \cup S)$.

From right to left: Assume that there are votes for the manipulators in $S$ that make $p$ the unique winner of $(C, V \cup S)$. Without loss of generality, assume that $p$ is on the first position in all votes

Table 3: Level $i$ scores in $(C, V \cup S)$ for $i \in\{1,2\}$ and the candidates in $C$

\begin{tabular}{lccccc}
\hline & $b$ & $c$ & $d$ & $p$ \\
\cline { 5 - 6 } score $^{1}$ & $3 K-1$ & & $3 K-1$ & 0 & $2 K$ \\
score $^{2}$ & $4 K-1$ & & $4 K-1$ & $4 K-2$ & $4 K$ \\
\hline
\end{tabular}


in $S$. Note that $p$ cannot win the manipulated election on the first level, so $p$ has to be the unique level 2 winner with score ${ }_{(C, V \cup S)}^{2}(p)=4 K$. This implies that $\operatorname{score}_{(C, V \cup S)}^{2}(e)<4 K$ has to hold for all $e \in\{b, c, d\}$. Since $a_{i} \geq 2$, candidate $d$ cannot be on the second position in any manipulator's vote. Thus, the manipulators' votes can be only of the form $(p>c>b>d),(p>c>d>b)$, $(p>b>c>d)$, or $(p>b>d>c)$. The candidates $b$ and $c$ have already $3 K-1$ points on the second level in $(C, V)$, so they each cannot gain more than $K$ points on the second level from the votes in $S$. Since all votes in $S$ have either $b$ or $c$ on the second position, the weights of the manipulators have to be of the form that a subset $A^{\prime} \subseteq A$ can be found such that those manipulators with weight $a_{i}, i \in A^{\prime}$, have a total weight of $K$ and put one of $b$ and $c$ (say $b$ ) on the second position, and the remaining manipulators (those with weight $a_{i}$ for $i \notin A^{\prime}$ ) put the other candidate, $c$, on the second position and have a total weight of $K$ as well. Thus, $\left(A,\left(a_{1}, a_{2}, \ldots, a_{k}\right)\right)$ is a yes-instance of PARTITION.

We now turn to the desctructive variant of coalitional weighted manipulation and give a deterministic polynomial-time algorithm for this problem in Bucklin voting.

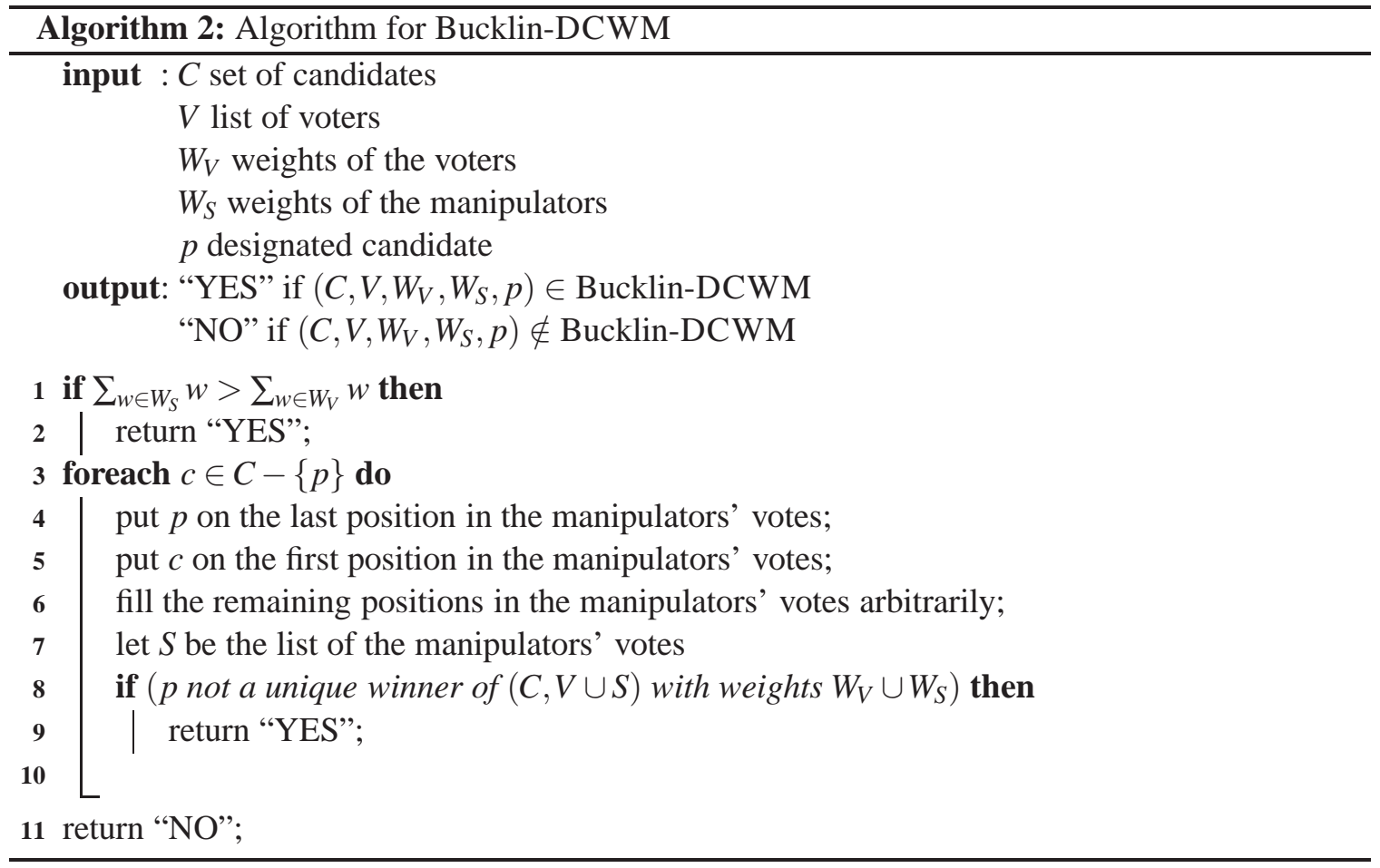

Before proving the runtime and correctness of the above algorithm, we state the following useful lemma, which is easily seen to hold.

Lemma 3.8 Let $(C, V)$ be a weighted Bucklin election with weights $W$ and $c, p \in C$. Then the following holds.

1. Assume that $c$ is not a winner in $(C, V)$ and that the votes in $V$ are changed in a way such that only the position of $c$ is made worse. Then $c$ is still not a winner. 
2. Assume that $c$ is a winner of the election and that the votes in $V$ are changed in a way such that only the position of $c$ is improved. Then c remains a winner.

3. Assume that $c$ is a winner of the election and that $p$ is not a winner. If in some votes the positions of candidates are swapped without changing the positions of $c$ and $p$, then $p$ is still not a winner.

We now analyze Algorithm $\underline{2}$ for Bucklin-DCWM.

Theorem 3.9 Algorithm $\underline{2}$ has a runtime in $\mathscr{O}\left(m^{2}\left(n+\left\|W_{S}\right\|\right)\right)$ and decides Bucklin-DCWM.

Proof. We begin with analyzing the runtime. Obviously, the algorithm always terminates and the input size is in $\mathscr{O}(\underbrace{m}_{\|C\|}+\underbrace{n m}_{\|V\|}+\underbrace{n}_{\left\|W_{V}\right\|}+\left\|W_{S}\right\|+\underbrace{1}_{\|p\|})=\mathscr{O}\left(n m+\left\|W_{S}\right\|\right)$.

The most costly part of the algorithm is the for-loop. To construct the manipulators' votes, $\mathscr{O}\left(\left\|W_{S}\right\| m\right)$ steps are needed. The winner-determination procedure for Bucklin can be implemented with a runtime of $\mathscr{O}(\mathrm{nm})$, so the if-statement in line 8 can be computed in time $\mathscr{O}\left(m\left(n+\left\|W_{S}\right\|\right)\right.$. Thus, the whole for-loop runs in time $\mathscr{O}\left(m^{2}\left(n+\left\|W_{S}\right\|\right)\right)$.

To prove the correctnes of the algorithm, we show that it gives the output "YES" if and only if $\left(C, V, W_{V}, W_{S}, p\right) \in$ Bucklin-DCWM.

From left to right: If the algorithm outputs "YES" in line 2 then we have $\sum_{w \in W_{S}} w>\sum_{w \in W_{V}} w$, i.e., the sum of the manipulators' weights is greater than the sum of the weights of the nonmanipulative voters. In this case, any of the candidates $c \neq p$ can be made the unique level 1 Bucklin winner in $(C, V \cup S)$ by putting $c$ on the first position in all the manipulators' votes and filling the remaining positions arbitrarily. Hence, $\left(C, V, W_{V}, W_{S}, p\right) \in$ Bucklin-DCWM. If the algorithm outputs "YES" in line 9, the manipulators' votes have been constructed such that $p$ is not a unique winner in $(C, V \cup S)$. Thus, we have that $\left(C, V, W_{V}, W_{S}, p\right)$ is a yes-instance of Bucklin-DCWM.

From right to left: Assume that $\left(C, V, W_{V}, W_{S}, p\right) \in$ Bucklin-DCWM. If $\sum_{w \in W_{S}} w>\sum_{w \in W_{V}} w$, then the algorithm correctly outputs "YES." Otherwise, the following holds: Since the given instance is a yes-instance of Bucklin-DCWM, the votes of the manipulators in $S$ can be set such that $p$ is not a winner of the election $(C, V \cup S)$. We know from Lemma $\underline{3.8}$ that successively swapping $p$ with her neighbor until $p$ is on the last position in all votes in $S$ does not change the fact that $p$ is not a winner in $\left(C, V \cup S^{\prime}\right)$ (where $S^{\prime}$ are the new manipulative votes with $p$ on the last position). Assume that $c \in C-\{p\}$ is a winner in $(C, V \cup S)$. Then swap her position successively with her neighbor in the votes in $S^{\prime}$ until $c$ is on the first position in all manipulative votes. Let $S^{\prime \prime}$ denote the accordingly changed list of manipulative votes. Again, from Lemma 3.8 we know that $c$ still wins in $\left(C, V \cup S^{\prime \prime}\right)$. Let $S^{\prime \prime \prime}$ be the list of manipulative votes that the algorithm constructs. We can transform $S^{\prime \prime}$ into $S^{\prime \prime \prime}$ by swapping the corresponding candidates $c^{\prime}, c^{\prime \prime} \in C-\{c, p\}$ accordingly. Since the positions of $c$ and $p$ remain unchanged, we have with Lemma $\underline{3.8}$ that $p$ is still not a winner in $\left(C, V \cup S^{\prime \prime \prime}\right)$. Thus, the algorithm outputs "YES" in line 9. 


\section{Bribery in Bucklin and Fallback Voting}

\subsection{Definition of Bribery Problems and Overview of Results}

We begin with defining the standard bribery scenarios proposed by Faliszewski et al. [FHH09] (see also [FHHR09]) that will be applied here to fallback and Bucklin elections. Let $\mathscr{E}$ be a given election system.

\begin{tabular}{ll}
\hline \multicolumn{1}{c}{$\mathscr{E}$-CONSTRUCTIVE UnweIGHTED BRIBERY $(\mathscr{E}$-CUB $)$} \\
\hline Given: & An $\mathscr{E}$ election $(C, V)$, a designated candidate $p$, and a nonnegative integer $k$. \\
Question: & $\begin{array}{l}\text { Is it possible to make } p \text { the unique } \mathscr{E} \text { winner by changing the votes of at most } k \\
\text { voters? }\end{array}$ \\
\hline
\end{tabular}

This basic bribery scenario can be extended by either considering voters with different weights, or allowing that each voter has a different price for changing her vote, or both. These three scenarios are formally defined by the following problems:

\begin{tabular}{|c|c|}
\hline \multicolumn{2}{|r|}{$\mathscr{E}$-Constructive Weighted Bribery $(\mathscr{E}$-CWB $)$} \\
\hline Given: & $\begin{array}{l}\text { An } \mathscr{E} \text { election }(C, V) \text { with each voter } v_{i} \in V \text { having a nonnegative integer } \\
\text { weight } w_{i} \text {, a designated candidate } p \text {, and a positive integer } k \text {. }\end{array}$ \\
\hline Question: & $\begin{array}{l}\text { Is it possible to make } p \text { the unique } \mathscr{E} \text { winner by changing the votes of at most } k \\
\text { voters? }\end{array}$ \\
\hline & 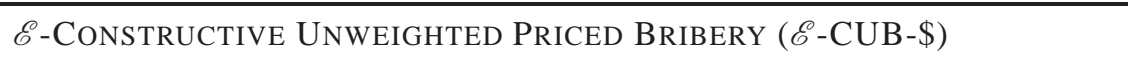 \\
\hline Given: & $\begin{array}{l}\text { An } \mathscr{E} \text { election }(C, V) \text { with each voter } v_{i} \in V \text { having a nonnegative integer price } \pi_{i} \text {, } \\
1 \leq i \leq n \text {, a designated candidate } p \text {, and a positive integer } k \text {. }\end{array}$ \\
\hline Question: & $\begin{array}{l}\text { Is there a set } B \subseteq\{1, \ldots, n\} \text { such that } \sum_{i \in B} \pi_{i} \leq k \text { and the voters } v_{i} \text { with } i \in B \text { can } \\
\text { be bribed so that } p \text { is the unique } \mathscr{E} \text { winner in the resulting election? }\end{array}$ \\
\hline & $\mathscr{E}$-Constructive Weighted Priced Bribery $(\mathscr{E}-\mathrm{CWB}-\$)$ \\
\hline Given: & $\begin{array}{l}\text { An } \mathscr{E} \text { election }(C, V) \text { with each voter } v_{i} \in V \text { having nonnegative integer weight } \\
w_{i} \text { and price } \pi_{i}, 1 \leq i \leq n, \text { a designated candidate } p \text {, and a positive integer } k \text {. }\end{array}$ \\
\hline Question: & $\begin{array}{l}\text { Is there a set } B \subseteq\{1, \ldots, n\} \text { such that } \sum_{i \in B} \pi_{i} \leq k \text { and the voters } v_{i} \text { with } i \in B \text { can } \\
\text { be bribed so that } p \text { is the unique } \mathscr{E} \text { winner in the resulting election? }\end{array}$ \\
\hline
\end{tabular}

By changing the question in the above four problems to ask whether $p$ can be prevented from being a unique winner of the election by bribing some of the voters, we obtain the destructive variants of these bribery scenarios, and we denote the corresponding problems by $\mathscr{E}$-DUB, $\mathscr{E}$ DWB, $\mathscr{E}$-DUB-\$, and $\mathscr{E}$-DWB-\$. The problems related to the general bribery scenarios without explicitly specifying the constructive or destructive case are denoted by $\mathscr{E}-\mathrm{UB}, \mathscr{E}-\mathrm{WB}, \mathscr{E}-\mathrm{UB}-\$$, and $\mathscr{E}-\mathrm{WB}-\$$.

Table $\underline{4}$ gives an overview of our complexity results for bribery in Bucklin and fallback voting. 
Table 4: Overview of results for bribery in Bucklin and fallback voting

\begin{tabular}{|c|c|c|c|c|}
\hline & \multicolumn{2}{|c|}{ Bucklin voting } & \multicolumn{2}{|c|}{ fallback voting } \\
\hline & complexity & reference & complexity & reference \\
\hline $\mathscr{E}$-CUB & $\mathrm{NP}-$ & Thn & $\mathrm{NF}$ & Th \\
\hline $\mathscr{E}$-DUB & $\mathrm{P}$ & Cor. $\underline{4.6}$ & $\mathrm{P}$ & Thm. $\underline{4.7}$ \\
\hline $\mathscr{E}$-CUB-\$ & NP-complete & Cor. $\underline{4.2}$ & NP-complete & Cor. $\underline{4.4}$ \\
\hline $\mathscr{E}$-DUB-\$ & $\mathrm{P}$ & Thm. 4.5 & $\mathrm{P}$ & Thm. $\underline{4.7}$ \\
\hline $\mathscr{E}-\mathrm{CWB}$ & NP-complete & Cor. $\underline{4.2}$ & NP-complete & Cor. $\underline{4.4}$ \\
\hline $\mathscr{E}$-DWB & $\mathrm{P}$ & Thm. 4.5 & $\mathrm{P}$ & Thm. 4.7 \\
\hline $\mathscr{E}-\mathrm{CWB}-\$$ & NP-con & Cor. $\underline{4.2}$ & NP-col & Cor. $\underline{4.4}$ \\
\hline $\mathscr{E}$-DWB-\$ & NP-complete & Thm. $\underline{4.8}$ & NP-complete & Thm. $\underline{4.9}$ \\
\hline
\end{tabular}

\subsection{Results for Bribery}

We start with the constructive cases of the standard bribery scenarios.

Theorem 4.1 CUB is NP-complete for Bucklin voting.

Proof. Membership of Bucklin-CUB in NP is obvious. We show NP-hardness by a reduction from Exact Cover By Three-Sets (X3C): Given a set $B=\left\{b_{1}, b_{2}, \ldots, b_{3 m}\right\}, m \geq 1$, and a collection $\mathscr{S}=\left\{S_{1}, S_{2}, \ldots, S_{n}\right\}$ of subsets $S_{i} \subseteq B$ with $\left\|S_{i}\right\|=3$ for each $i, 1 \leq i \leq n$, is there a subcollection $\mathscr{S}^{\prime} \subseteq \mathscr{S}$ such that each element of $B$ occurs in exactly one set in $\mathscr{S}^{\prime}$ ? X3C is a well-known NP-complete problem (see, e.g., [GJ79]).

Let $(B, \mathscr{S})$ be an instance of X3C with $B=\left\{b_{1}, b_{2}, \ldots, b_{3 m}\right\}$ and $\mathscr{S}=\left\{S_{1}, S_{2}, \ldots, S_{n}\right\}$. Without loss of generality, we may assume that $n \geq 2 m$. We construct a Bucklin-CUB instance $((C, V), p, k)$, where $(C, V)$ is a Bucklin election with the candidates $C=B \cup\{c, d\} \cup G \cup\{p\}, p$ is the designated candidate, and $k=m$. $G$ is a set of "padding candidates," which are used to ensure that certain candidates do not gain points up to a certain level. Padding candidates are positioned in the votes such that, up to a certain level, they do not gain enough points (e.g., at most one) to be relevant for the central argumentation of the proof. Thus, their scores are not listed in tables giving the scores of the relevant candidates.

For every $b_{j} \in B$, define $\ell_{j}$ to be the number of sets $S_{i} \in \mathscr{S}$ candidate $b_{j}$ is contained in. $V$ consists of the following $2 n$ voters (i.e., a strict majority is reached with $n+1$ votes):

- The first voter group consists of $n$ voters. For each $i, 1 \leq i \leq n$, we have one voter of the form

$$
c>d>S_{i}>G_{1}>\left\{C-\left(\{c, d\} \cup S_{i} \cup G_{1}\right)\right\},
$$

where $G_{1} \subseteq G$ is a set of $3 m-3$ padding candidates. When a set $X$ of candidates is giving in such a ranking, the order of the candidates from $X$ can be fixed arbitrarily in this ranking.

- The second voter group consists of $n$ voters as well. We will present the preferences level by level from the first to the $(3 m+2)$ nd position in Table $\underline{5}$.

Note that the padding candidates in $G$ are positioned in the votes such that every $g_{k} \in G$ gains at most one point up to level $3 m+2$. Table $\underline{6(a)}$ shows the scores of the relevant candidates in $(C, V)$ 
Table 5: Construction of Bucklin election $(C, V)$ in the proof of Theorem $\underline{4.1}$

\begin{tabular}{|c|c|c|}
\hline position & \# voters & candidate \\
\hline \multirow{3}{*}{1} & $m$ & $c$ \\
\hline & & $d$ \\
\hline & $n-2 m$ & $g_{k}$ \\
\hline \multirow{2}{*}{2} & $n+1-\ell_{1}$ & $b_{1}$ \\
\hline & $\ell_{1}-1$ & from $G_{2}$ \\
\hline \multirow{2}{*}{3} & $n+1-\ell_{2}$ & $b_{2}$ \\
\hline & $\ell_{2}-1$ & from $G_{2}$ \\
\hline & $\vdots$ & $\vdots$ \\
\hline \multirow[t]{2}{*}{$3 m+1$} & $n+1-\ell_{3 n}$ & $b_{3 m}$ \\
\hline & $\ell_{3 n}-1$ & from $G_{2}$ \\
\hline \multirow[t]{2}{*}{$3 m+2$} & $n-m+1$ & $p$ \\
\hline & $m-1$ & from $G_{2}$ \\
\hline
\end{tabular}

Table 6: Level $i$ scores for $i \in\{1,2,3 m, 3 m+1,3 m+2\}$ and the candidates in $C-G$

(a) Original election $(C, V)$

\begin{tabular}{|c|c|c|c|c|}
\hline & $b_{i} \in B$ & $c$ & $d$ & $p$ \\
\hline score $^{1}$ & 0 & $n+m$ & $m$ & 0 \\
\hline score $^{2}$ & $\leq n+1$ & $n+m$ & $m+n$ & 0 \\
\hline score $^{3 m}$ & $\leq n+1$ & $n+m$ & $m+n$ & 0 \\
\hline $\operatorname{score}^{3 m+1}$ & $\leq n+1$ & $n+m$ & $m+n$ & 0 \\
\hline $\operatorname{score}^{3 m+2}$ & $n+1$ & $n+m$ & $m+n$ & $n-m+1$ \\
\hline
\end{tabular}

(b) Modified election $\left(C, V^{\prime}\right)$

\begin{tabular}{|c|c|c|c|c|}
\hline & $b_{i} \in B$ & $c$ & $d$ & $p$ \\
\hline score 1 & 0 & $\bar{n}$ & $m$ & $m$ \\
\hline score $^{2}$ & $\leq n$ & $n$ & $n$ & $m$ \\
\hline score $^{3 m}$ & $\leq n$ & $n$ & $n$ & $m$ \\
\hline $\operatorname{score}^{3 m+1}$ & $\leq n$ & $n$ & $n$ & $m$ \\
\hline score $^{3 m+2}$ & $\leq n$ & $n$ & $n$ & $n+1$ \\
\hline
\end{tabular}

(namely, $c, d, p$, and each $b_{j} \in B$ ) for the relevant levels (namely, 1, 2, 3m, 3m+1, and $3 m+2$ ) and, in particular, that $c$ is the unique level 1 Bucklin winner in $(C, V)$.

We claim that $\mathscr{S}$ has an exact cover $\mathscr{S}^{\prime}$ for $B$ if and only if $p$ can be made the unique Bucklin winner by changing at most $m$ votes in $V$.

From left to right: Let $\mathscr{S}^{\prime}$ be an exact cover for $B$ and let $I \subseteq\{1, \ldots, n\}$ be the set of indices of the $m$ elements in $\mathscr{S}^{\prime}$. To make $p$ the unique Bucklin winner, we only have to change votes in the first voter group: For each $i \in I$, change the corresponding vote

$$
c>d>S_{i}>G_{1}>\left\{C-\left(\{c, d\} \cup S_{i} \cup G_{1}\right)\right\}
$$

to

$$
p>G_{1}>g_{1}^{\prime}>g_{2}^{\prime}>g_{3}^{\prime}>g_{4}^{\prime}>\left\{C-\left(\left\{g_{1}^{\prime}, g_{2}^{\prime}, g_{3}^{\prime}, g_{4}^{\prime}, p\right\} \cup G_{1}\right)\right\},
$$

where each $g_{j}^{\prime}, 1 \leq j \leq 4$, is from $G$ but not in $G_{1}$. 
With these new votes, $c$ and $d$ both lose $m$ points on the first two levels from the first voter group and $p$ gains $m$ points on the first level. Every candidate $b_{i} \in B$ loses exactly one point on one of the levels 3, 4, or 5. The scores in the resulting election $\left(C, V^{\prime}\right)$ are shown in Table 6(b). As one can see, $p$ is the first candidate to reach a strict majority of $n+1$ votes (namely, on level $3 m+2$ ) and is thus the unique level $3 m+2$ Bucklin winner in the new election.

From right to left: Assume that $p$ is the unique Bucklin winner of the election $\left(C, V^{\prime}\right)$, where $V^{\prime}$ is the new voter list containing the $m$ changed votes. Since only $m$ votes can be changed and $p$ did not score any points prior to level $3 m+2$ in the original election, $p$ has to be a level $3 m+2$ Bucklin winner in $\left(C, V^{\prime}\right)$. Candidates $c$ and $d$ originally reach the majority threshold already on, respectively, the first and the second level, so all votes that can be changed must place $c$ and $d$ on the first two positions. The only votes doing so are those in the first voter group. Finally, to prevent the candidates in $B$ from reaching a strict majority on level $3 m+2$, each of the $3 m$ candidates has to lose at least one point by changing at most $m$ votes. This, again, can only be done by changing votes from the first voter group and there has to be an exact cover $\mathscr{S}^{\prime}$ for $B$ whose corresponding voters from the first voter group have to be changed.

The following corollary follows immediately from Theorem $\underline{4.1}$.

Corollary 4.2 In Bucklin elections, CWB, CUB-\$, and CWB-\$ are NP-complete.

Based on the corresponding proof for approval voting that is due to Faliszewski et al. [FHH09], we can show NP-completeness for unweighted bribery in fallback elections as well.

Theorem 4.3 CUB is NP-complete for fallback voting.

Proof. Fallback-CUB obviously is in NP. To show NP-hardness, we give a reduction from X3C. Let $(B, \mathscr{S})$ be an instance of X3C with $B=\left\{b_{1}, b_{2}, \ldots, b_{3 m}\right\}$ and $\mathscr{S}=\left\{S_{1}, S_{2}, \ldots, S_{n}\right\}$. We define the fallback election $(C, V)$ with the candidate set $C=B \cup E \cup\{p\}$, where $p$ is the designated candidate and $E$ is a set of $n+m$ padding candidates. For every $j \in\{1, \ldots, 3 m\}$, we define $\ell_{j}$ as the number of subsets $S_{i} \in \mathscr{S}$ candidate $b_{j} \in B$ is contained in. Using this notation, we define the subsets $B_{i}=\left\{b_{j} \in B \mid i \leq n-\ell_{j}\right\}$ for $i \in\{1, \ldots, n\}$. $V$ consists of the $4 n$ voters whose preferences are given in Table $\underline{7}$.

Table 7: Construction of fallback election $(C, V)$ in the proof of Theorem $\underline{4.3}$

\begin{tabular}{clcll}
\hline$\#$ & For each $\ldots$ & number of votes & & ranking of candidates \\
\cline { 2 - 2 }$\frac{i}{1}$ & $i \in\{1, \ldots, n\}$ & 1 & & $S_{i} \mid\left(B-S_{i}\right) \cup E \cup\{p\}$ \\
2 & $i \in\{1, \ldots, n\}$ & 1 & & $B_{i} \mid\left(B-B_{i}\right) \cup E \cup\{p\}$ \\
3 & & $n-m$ & & $p \mid B \cup E$ \\
4 & $\ell \in\{1, \ldots, n+m\}$ & 1 & & $e_{\ell} \mid B \cup\left(E-\left\{e_{\ell}\right\} \cup\{p\}\right.$ \\
\hline
\end{tabular}

In this election, we have that $\operatorname{score}(p)=n-m, \operatorname{score}\left(b_{j}\right)=n$ for all $j \in\{1, \ldots, 3 m\}$, and $\operatorname{score}\left(e_{\ell}\right)=1$ for all $\ell \in\{1, \ldots, n+m\}$. Since no candidate reaches a strict majority (at least $2 n+1$ points), all candidates $b_{j} \in B$ are fallback winners of this election. 
We claim that $\mathscr{S}$ has an exact cover $\mathscr{S}^{\prime}$ for $B$ if and only if $p$ can be made the unique fallback winner by bribing at most $m$ voters.

From left to right: Suppose that $\mathscr{S}$ has an exact cover $\mathscr{S}^{\prime}$ for $B$. We change the vote of those voters in the first voter group where $S_{i} \in \mathscr{S}^{\prime}$ from $S_{i} \mid\left(B-S_{i}\right) \cup E \cup\{p\}$ to $p \mid B \cup E$. In the resulting election $\left(C, V^{\prime}\right)$ only the scores of the candidates in $B$ and the score of $p$ change: $p$ gains $m$ points, whereas each $b_{j} \in B$ loses exactly one point. Thus, with an overall score of $n$, candidate $p$ is the unique fallback winner of the resulting election.

From right to left: Suppose that $p$ can be made the unique fallback winner by changing at most $m$ votes in $V$. That means that $p$ can gain at most $m$ points, so the maximum overall score that $p$ can reach is $n$. Since each $b_{j} \in B$ has an overall score of $n$, every candidate in $B$ has to lose at least one point by changing at most $m$ votes (otherwise, there would be at least one candidate in $B$ that ties with $p$ ). This is possible only if in $m$ votes of the first voter group the candidates in $S_{i}$ are removed from the approval strategy such that these $m$ sets $S_{i}$ form an exact cover for $B$.

This result immediately implies NP-hardness for the remaining constructive bribery scenarios in fallback elections as well.

Corollary 4.4 In fallback elections, CWB, CUB-\$, and CWB-\$are NP-complete.

We now turn to the destructive cases. The following result generalizes a result due to Xia [Xia12] who showed that DUB is in P for simplified Bucklin voting.

Theorem 4.5 In Bucklin elections, DWB and DUB-\$are in $\mathrm{P}$.

Proof. Both problems, Bucklin-DWB and Bucklin-DUB-\$, can be solved by deterministic polynomial-time algorithms that use Algorithm $\underline{2}$, which was designed in Section $\underline{3}$ to solve the destructive coalitional weighted manipulation problem for Bucklin elections, Bucklin-DCWM. The main difference between a bribery and a manipulation instance is that in the latter only the preferences of the manipulators have to be found, whereas in the former both the votes that will be bribed and the new preferences for these voters have to be found. If we have the set of votes we want to change, we can use the algorithm for the manipulation problem to construct the preferences. Thus, for the runtime of the algorithm the determination of these voter sets is crucial, and we show that in Bucklin elections the number of voter sets whose modification might actually lead to a successful bribery is bounded by a polynomial in both the number of voters and the number of candidates.

Consider Algorithm $\underline{3}$ and a given input $\left(C, V, W_{V}, p, k\right)$ to it. In particular, $p$ is the designated candidate that we want to prevent from winning and assume that we have a yes-instance, i.e., our bribery action is successful. We denote by $\left(C, V^{\prime \prime}\right)$ the election resulting from $(C, V)$ where the $k$ votes that can be changed have already been changed. Then there is a candidate $c \in C-\{p\}$ that reaches a strict majority on level $i$, and it holds that $\operatorname{score}_{\left(C, V^{\prime \prime}\right)}^{i}(c) \geq \operatorname{score}_{\left(C, V^{\prime \prime}\right)}^{i}(p)$, which means that $p$ is not a unique winner in $\left(C, V^{\prime \prime}\right)$. To reach that, for each $i<m$, there are only five types of preferences that might have been changed in $V$, and they can be grouped into the following subsets $T_{i, j} \subseteq V, 1 \leq j \leq 5$ :

$T_{i, 1}: p$ is among the top $i-1$ position and $c$ is among the top $i$ positions (when changing: $p$ loses points, $c$ does neither lose nor win points up to level $i$ ). 
$T_{i, 2}: p$ is among the top $i-1$ position and $c$ is not among the top $i$ positions (when changing: $p$ loses points, $c$ wins points up to level $i$ ).

$T_{i, 3}: p$ is on position $i$ and $c$ is among the top $i$ positions (when changing: $p$ loses points, $c$ does neither lose nor win points up to level $i$ ).

$T_{i, 4}: p$ is on position $i$ and $c$ is not among the top $i$ positions (when changing: $p$ loses points, $c$ wins points up to level $i$ ).

$T_{i, 5}$ : both $p$ and $c$ are not among the top $i$ positions (when changing: $p$ does neither lose nor win points, $c$ wins points up to level $i$ ).

For a sublist of voters $V^{\prime} \subseteq V$, denote their total weight by $W_{V}^{\prime}$. Algorithm $\underline{3}$ for Bucklin-DWB works as follows.

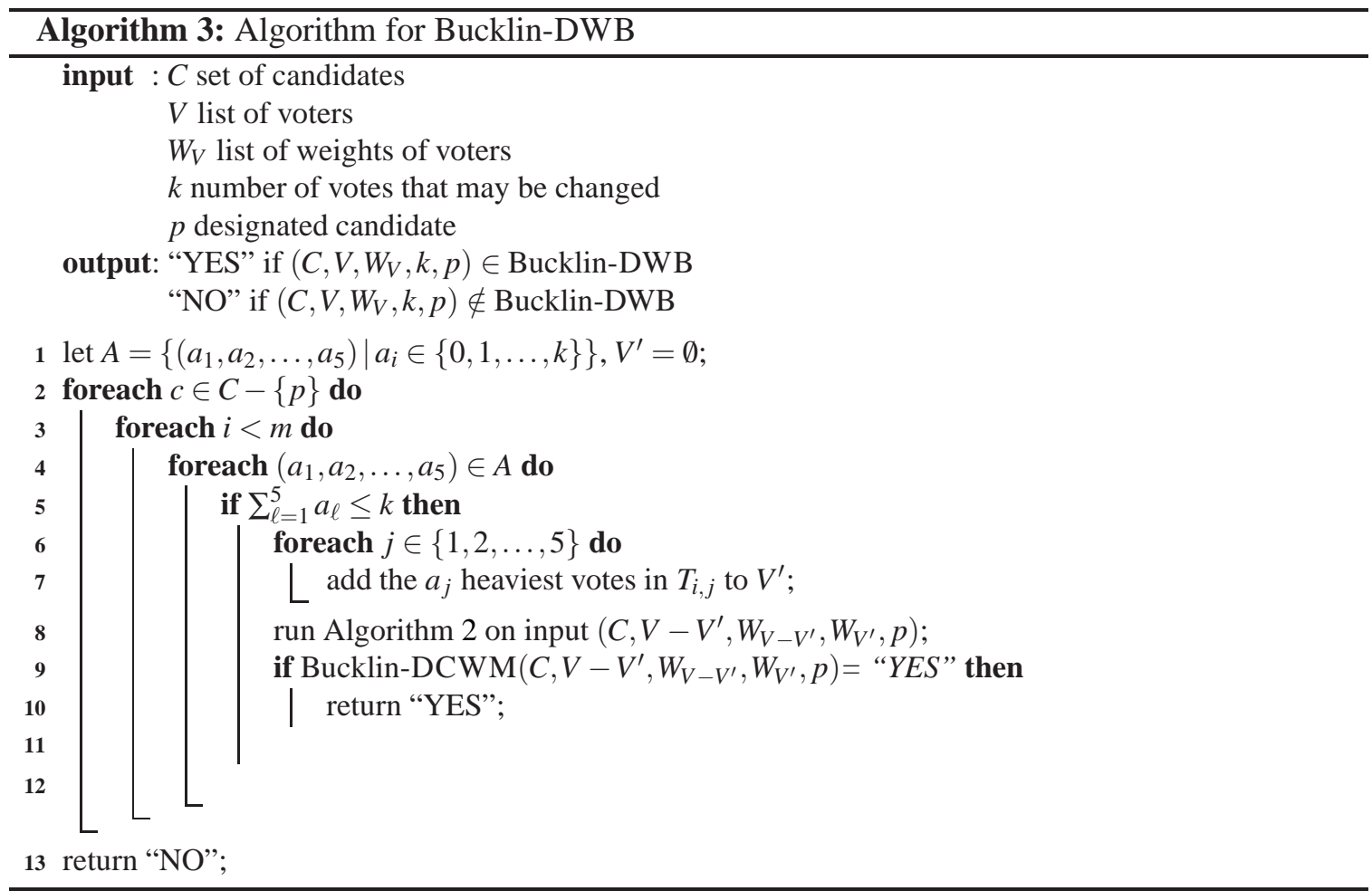

It is easy to see that Algorithm $\underline{3}$ runs in deterministic polynomial time: the two outer for-loops iterate up to $m$ times, whereas the inner loop tests up to $k^{5}$ variations of the vector $\left(a_{1}, a_{2}, \ldots, a_{5}\right)$. Since $k \leq n$, we have that the number of executions of Algorithm 2 is in $\mathscr{O}\left(m^{2} n^{5}\right)$.

For the proof of correctnes, we show that given a bribery instance $\left(C, V, W_{V}, k, p\right)$, the output of Algorithm $\underline{3}$ is "YES" if and only if $\left(C, V, W_{V}, k, p\right) \in$ Bucklin-DWB.

From left to right: If the algorithm returns "YES" in line 10, then Algorithm $\underline{2}$ could find a successful destructive manipulation regarding $p$ for $k$ manipulators with total weight $W_{V^{\prime}}$. So $p$ is not a unique Bucklin winner in the election $\left(C, V^{\prime \prime}\right)$, where $V^{\prime \prime}$ is the list of voters with $k$ changed votes. That means that $\left(C, V, W_{V}, k, p\right) \in$ Bucklin-DWB. 
From right to left: Assume that $\left(C, V, W_{V}, k, p\right) \in$ Bucklin-DWB. Thus, there exists a set of $k$ voters $V^{\prime}$ with total weight $W_{V^{\prime}}$ such that changing these votes prevents $p$ from being a unique Bucklin winner in $\left(C, V^{\prime \prime}\right)$, where $V^{\prime \prime}$ is the new voter list containing the $k$ changed votes. We want to show that such a $V^{\prime \prime}$ can always be transformed to the list of votes $V^{\prime}$ that is changed in Algorithm $\underline{3}$. From our assumptions it follows that we have a candidate $c \in C-\{p\}$ and a level $i<m$ such that $c$ is a level $i$ Bucklin winner that prevents $p$ from being a unique winner.

Assume that in $V^{\prime \prime}$ are voters whose preferences were not in one of the $T_{i}$ before the changes were made, i.e., votes were changed that not necessarily needed to be changed to prevent $p$ from being the only winner. Undo these changes and change the same number of votes in the lists $T_{i}$ that were not changed before. We then have that all changed votes are in one of the $T_{i}$.

Since Bucklin is monotonic, we can always exchange votes with higher weight with votes of lower weight (in one $T_{i}$ ) without risking that $p$ would win due to this exchange. So we know that we can transform any given list of bribed votes to a list that the algorithm would construct and this would still prevent $p$ from winning alone. So if there is a list of $k$ voters that can be successfully bribed to prevent $p$ from being the unique winner, the algorithm will find it.

For the Bucklin-DUB-\$ problem the same algorithm can be used. The only difference is that all weights have to be set to one, the cheapest instead of the heaviest votes (i.e., those votes with the least price instead of the greatest weight) are added to $V^{\prime}$ in line 7, and it has to be tested whether the sum of the chosen votes does not exceed the budget.

From Theorem $\underline{4.5}$ we have the following corollary.

Corollary 4.6 In Bucklin elections, DUB is in P.

This algorithm can be easily adapted for fallback elections. Due to the fact that in fallback elections the voters do not have to rank all candidates, it is possible that a candidate wins on level $m$. So by making the following changes in Algorithm $\underline{2}$ :

- change " $i<m$ " in line 3 to " $i \leq m$,"

- use the fallback analogue of Algorithm $\underline{2}$ in line $8,{ }_{-}^{4}$ and

- change "Bucklin-DCWM" in line 9 to "Fallback-DCWM,"

we can decide DWB for fallback elections as well.

Theorem 4.7 In fallback elections, DWB, DUB, and DUB-\$are in $\mathrm{P}$.

It remains to show the complexity of the destructive variant of priced bribery in weighted Bucklin and fallback elections. We begin with showing NP-hardness of this problem for Bucklin voting.

Theorem 4.8 Bucklin-DWB-\$ is NP-complete.

\footnotetext{
${ }^{4}$ In Section 3.3 , we refrained from explicitly stating the algorithm for fallback-DCWM that is based on the following simple idea: For every candidate $c \neq p$, try to make $c$ win by setting the manipulators' votes to $c \mid C-\{c\}$. If such a candidate can be found, $p$ has been successfully prevented from being the unique winner of the election; otherwise, it is impossible to do so.
} 
Proof. That Bucklin-DWB-\$ is in NP is again easy to see. We show NP-hardness by a reduction from PARtition. Let $\left(A,\left(a_{1}, \ldots, a_{k}\right)\right)$ with $A=\{1, \ldots, k\}$ and $\sum_{i=1}^{k} a_{i}=2 K$ be an instance of PARTition. We construct the following Bucklin election $(C, V)$ with $C=\{c, p\}$ and $k$ votes in $V$ : For each $i \in\{1, \ldots, k\}$, we have one voter with weight $w_{i}=a_{i}$, price $\pi_{i}=a_{i}$, and preference $p>c$.

The total weight in $(C, V)$ is $2 K$. Let $K$ be the budget that may not be exceeded and let $p$ be the designated candidate. Obviously, $p$ is the unique level 1 Bucklin winner in $(C, V)$.

We claim that $\left(A,\left(a_{1}, \ldots, a_{k}\right)\right) \in$ PARTITION if and only if $p$ can be prevented from being the unique Bucklin winner by changing votes in $V$ without exceeding the budget $K$.

From left to right: Let $\left(A,\left(a_{1}, \ldots, a_{k}\right)\right) \in$ PARTITION with $A^{\prime} \subseteq A$ such that $\sum_{i \in A^{\prime}} a_{i}=K$. Change the votes of those voters with $w_{i}=a_{i}$ for $i \in A^{\prime}$ from $p>c$ to $c>p$. With these changes we have that on the first level, both $p$ and $c$ have exactly $K$ points, so no strict majority. On the second level, both candidates have $2 K$ points and thus are both level 2-Bucklin winners. Hence, $p$ is not a unique Bucklin winner in the bribed election.

From right to left: Assume that $p$ is not a unique Bucklin winner in the bribed election. Since there are only two levels, either $c$ is the unique level 1 winner, or $p$ and $c$ both win on the second level. The price and weight of every voter is the same, so voters with a total weight of $K$ can be changed. Candidate $c$ has 0 points in the original election, so it is not possible to make $c$ a unique level 1 winner without exceeding the budget $K$. To prevent $p$ from remaining the unique winner on the first level, the budget has to be fully exhausted and votes with a total weight of $K$ must be changed from $p>c$ to $c>p$. Thus, there is a subset $A^{\prime} \subseteq A$ such that $\sum_{i \in A^{\prime}} a_{i}=K$, so $\left(A,\left(a_{1}, \ldots, a_{k}\right)\right) \in$ PARTITION.

Theorem $\underline{4.9}$ states the corresponding result for fallback voting using a similar proof idea.

Theorem 4.9 Fallback-DWB-\$ is NP-complete.

Proof. Obviously, fallback-DWB- $\$$ is in NP. NP-hardness is shown by a reduction from PARTITION. To this end, let $\left(A,\left(a_{1}, \ldots, a_{k}\right)\right)$ with $A=\{1, \ldots, k\}$ and $\sum_{i=1}^{k} a_{i}=2 K$ be an instance of PARTition. We construct a fallback election $(C, V)$ with the candidate set $C=\{c, p\}$ and the designated candidate $p$. Let $V$ consist of $k$ voters $v_{1}, \ldots, v_{k}$, each having preference $p \mid\{c\}$, weight $w_{i}=a_{i}$, and price $\pi_{i}=a_{i}$. The total weight in $(C, V)$ is $2 K$ and $p$ is the unique fallback winner in this election. Let the briber's budget be $K$.

We claim that $\left(A,\left(a_{1}, \ldots, a_{k}\right)\right) \in$ PARTITION if and only if $p$ can be prevented from being the unique fallback winner by changing votes in $V$ without exceeding the budget $K$.

From left to right: Assume that there is a set $A^{\prime} \subseteq A$ such that $\sum_{i \in A^{\prime}} a_{i}=K$. We change the votes of each voter $v_{i}$ with $i \in A^{\prime}$ from $p \mid\{c\}$ to $c \mid\{p\}$. Then both candidates, $p$ and $c$, have an overall score of $K$ and are both fallback winners of the resulting election.

From right to left: Assume that $p$ can be prevented from being the unique fallback winner by changing votes in $V$ without exceeding the budget $K$. This is possible only if candidate $c$ at least ties with $p$ after the changes in the votes have been made. Since each voter's weight and price are the same, $c$ can gain at most $K$ points without exceeding the budget $K$. To prevent $p$ from being the unique fallback winner, $c$ has to gain at least $K$ points, so together with the budget restriction $c$ has to gain exactly $K$ points. Thus, there has to be a set $A^{\prime} \subseteq A$ such that $\sum_{i \in A^{\prime}} a_{i}=K$. 


\section{Campaign Management}

In the discussion so far, we have focused on bribery and manipulation as means of attacking Bucklin and fallback elections. However, it is also quite natural to consider bribery scenarios through the lenses of running a political campaign. After all, in a successful campaign, the candidates spend their effort (measured in terms of time, in terms of financial cost of organizing promotional activities, and even in terms of the difficulty of convincing particular voters) to change the minds of the voters. Thus, formally, a campaign preceding an election can be seen as exchanging some resources for voters' support. Formally, this idea is very close to bribery (indeed, this view of campaign management was first presented in a paper whose focus was on a bribery problem [EFS09]).

\subsection{Definitions and Overview of Results}

We start by discussing one of the most general campaign management problems, namely the SWAP BRIBERY problem introduced by Elkind et al. [EFS09]. This problem models a situation where a campaign manager, who is interested in the victory of a given candidate $p$, can organize meetings with specific voters (the unweighted variant of the problem) or with groups of like-minded voters (the weighted variant) and convince them to change their preference orders. However, the difficulty (or, as we will say from now on, the cost) of changing the voters' preference orders depends both on the voter and on the extent of the change (for example, it might be expensive to swap a voter's most preferred candidate with this voter's least preferred one, but it might be very cheap to swap the voter's two least preferred candidates). Formally, Elkind et al. [EFS09] define so-called swapbribery price functions that for each voter and for each pair of candidates give the cost of swapping these two candidates in the voter's preference order (provided the candidates are adjacent in this order).

Definition 5.1 (Elkind et al. [EFS09]) A swap-bribery price function for voter $v_{i}$ is a function $\pi_{i}$ : $C \times C \rightarrow \mathbb{N}$ that specifies for any ordered pair $\left(c_{i}, c_{j}\right)$ of candidates the price for changing $v_{i}$ 's preference order from $\cdots>c_{i}>c_{j}>\cdots$ to $\cdots>c_{j}>c_{i}>\cdots$. Only candidates that are adjacent in a vote can be swapped.

In the $\mathscr{E}$-CONSTRUCTIVE SWAP BRIBERY problem we ask if there exists a sequence of swaps of adjacent candidates that lead to a given candidate being a winner (note that the swaps are performed in sequence; even if some candidates are not adjacent at first, they may become adjacent in the course of performing the swaps and, then, can be swapped themselves). .5

\begin{tabular}{ll}
\hline & $\mathscr{E}$-CONSTRUCTIVE UNweIGHTED SwAP BRIBERY $(\mathscr{E}$-CUSB) \\
\hline Given: & $\begin{array}{l}\text { An } \mathscr{E} \text {-election }(C, V), \text { where } V=\left(v_{1}, \ldots, v_{n}\right), \text { a designated candidate } p \text {, a list } \\
\left(\pi_{1}, \ldots, \pi_{n}\right) \text { of swap bribery price functions, and a nonnegative integer } k .\end{array}$ \\
Question: & $\begin{array}{l}\text { Can } p \text { be made the unique } \mathscr{E} \text { winner of an election resulting from the input elec- } \\
\text { tion by conducting a sequence of swaps of adjacent candidates in the voters' } \\
\text { ballots such that the total cost of the swaps does not exceed the budget } k ?\end{array}$ \\
\hline
\end{tabular}

\footnotetext{
${ }^{5}$ We mention that Elkind et al. [EFS09] defined the problem for the nonunique-winner model. Here we adopt the unique-winner model to stay in sync with the rest of the paper. However, it will be easy to see that all the results from this section hold in the nonunique-winner model as well.
} 
We define the weighted variant of the problem, $\mathscr{E}-\mathrm{CWSB}$, in the standard way (as far as we can tell, the weighted variant of the problem has not been studied before). However, it will soon become clear why the weighted variant is not particularly interesting and so we omit the easy modification of the definition. We also define the destructive variants of the swap bribery problems $(\mathscr{E}$-DUSB- $\$$ and $\mathscr{E}$-DWSB-\$) in the usual way, by changing the question to ask whether $p$ can be prevented from being a unique winner.

Swap bribery is a very difficult problem-it is NP-complete for almost all natural voting rules (and, in particular, in the next section we will see a very strong hardness result for the Bucklin and fallback rules). Thus Elkind et al. [EFS09] defined its much-simplified variant, shift-bribery, where every swap has to involve the designated candidate $p$ (that is, the designated candidate can be "shifted" forward in selected votes). The complexity of this problem was studied for a number of voting rules [EFS09, EF10a, DS12], including Bucklin and fallback voting [SFE11]. Interestingly, even though we will see strong hardness results for swap bribery under Bucklin and fallback, shift bribery for these rules is in $\mathrm{P}$.

The definitions of swap bribery and shift bribery are very natural for voting rules where each voter ranks all the candidates; for the case of fallback, where the ballots consist of the approved part (where the candidates are ranked) and of the disapproved part (where the candidates are not ranked), we need to extend the definitions. In our approach, we define swap bribery under fallback to allow the swaps within the approved parts of the votes only. Naturally, one could also define costs for including given disapproved candidates in the approved part and, indeed, Elkind et al. [EFS09] did so for SP-AV (SP-AV is a variant of the approval system). - However, following Schlotter et al. [SFE11], we believe that it is more informative to study the complexity of modifying the rankings within the approved parts and the complexity of modifying the sets of approved candidates separately.

Regarding the latter type of problems, Schlotter et al. [SFE11] defined the support bribery problem for the fallback rule (and other hybrid rules), where each voter has a complete preference order over the whole set of candidates, but also has an approval threshold, a number of top candidates that this voter approves of. For each voter we have a price function that gives the cost of increasing/decreasing the approval threshold; the goal is to change the voters' approval thresholds in such a way as to ensure the victory of a given candidate. Schlotter et al. [SFE11] show that this problem is NP-complete for fallback. ${ }_{-}$However, in our model the disapproved candidates are not ranked and, thus, it is much more natural to study the extension bribery problem introduced by Baumeister et al. [BFLR12]. The idea of extension bribery is to capture very non-invasive campaign actions, where we try to convince some voters to include the designated candidate at the end of the ranking of approved candidates.

Definition 5.2 (Baumeister et al. [BFLR12]) The extension bribery price function $\delta_{i}: \mathbb{N} \rightarrow \mathbb{N}$ of $a$ voter $v_{i}$ defines the price for extending the approved part of $v_{i}$ 's vote with a given number of

\footnotetext{
${ }^{6}$ Like fallback voting, SP-AV is a hybrid variant of approval voting. It has been introduced by Brams and Sanver [BS06] and slightly modified by Erdélyi et al. [ENR09] to cope with certain control actions (see also the chapter by Baumeister et al. $\left[\mathrm{BEH}^{+} 10\right]$ for a through discussion of this voting system).

${ }^{7}$ They also show that the problem is hard in the sense of parametrized complexity for two natural parameters describing the extent of change to the approval thresholds. Interestingly, they show the problem to be fixed-parameter tractable if the thresholds can either only increase or only decrease.
} 
Table 8: Overview of results for swap bribery and extension bribery in Bucklin and fallback voting

\begin{tabular}{|c|c|c|c|c|}
\hline & \multicolumn{2}{|c|}{ Bucklin voting } & \multicolumn{2}{|c|}{ fallback voting } \\
\hline & complexity & reference & complexity & reference \\
\hline $\mathscr{E}$-CUSB & NP-complete & Thm. 5.4 & NP-complete & Cor. $\underline{5.5}$ \\
\hline $\mathscr{E}$-DUSB & NP-complete & Cor. 5.5 & NP-complete & Cor. 5.5 \\
\hline $\mathscr{E}-\mathrm{CWSB}$ & NP-complete & Thm. $\underline{5.3}$ & NP-complete & Thm. $\underline{5.3}$ \\
\hline $\mathscr{E}$-DWSB & NP-complete & Thm. $\overline{5.3}$ & NP-complete & Thm. 5.3 \\
\hline $\mathscr{E}$-CUEB & - & & $\mathrm{P}$ & Thm. $\overline{5.8}$ \\
\hline $\mathscr{E}$-DUEB & - & & $\mathrm{P}$ & Thm. 5.8 \\
\hline $\mathscr{E}$-CWEB & - & & NP-complete & Thm. 5.7 \\
\hline $\mathscr{E}$-DWEB & - & & NP-complete & Thm. 5.7 \\
\hline
\end{tabular}

so-far-disapproved candidates (these new candidates are ranked below the previously-approved candidates, but among themselves are ranked as the briber requests).

We define the following related problem.

\begin{tabular}{cl}
\hline FV-CONSTRUCTIVE UNWEIGHTED EXTENSION BRIBERY (FV-CUEB) \\
\hline Given: $\quad \begin{array}{l}\text { A fallback election }(C, V) \text {, where } V=\left(v_{1}, \ldots, v_{n}\right), \text { a designated candidate } p \text {, a } \\
\text { list }\left(\delta_{1}, \ldots, \delta_{n}\right) \text { of extension bribery price functions, and a nonnegative integer } k .\end{array}$ \\
Question: $\quad \begin{array}{l}\text { Can } p \text { be made the unique fallback winner by extending the approved parts of } \\
\text { the the voters' ballots without exceeding the budget } k ?\end{array}$ \\
\hline
\end{tabular}

Again, the weighted variant $(\mathscr{E}-\mathrm{CWEB})$ is defined in the natural way and so are the destructive variants $(\mathscr{E}$-DUEB and $\mathscr{E}$-DWEB).

Table $\underline{8}$ summarizes the results of this section.

\subsection{Results for Swap Bribery}

We start by quickly observing that weighted swap bribery is NP-complete for both Bucklin and fallback rules.

\section{Theorem 5.3 BV-CWSB, BV-DWSB, FV-CWSB, and FV-DWSB are NP-complete.}

Proof. The proof for Bucklin is a direct consequence of the fact that CWB- $\$$ is NP-complete for plurality, even for just two candidates [FHH09] (the result holds both for the uniqe-winner case and for the nonunique-winner case). For two candidates, the Bucklin rule is identical to the plurality rule. Further, for two candidates CWB- $\$$ is, in essence, identical to CWSB (the only possible bribery is to swap the only two candidates), and the nonunique-winner variant of CWB-\$is, in essence, identical to DWSB.

For fallback, membership of the problems in NP is clear, and NP-hardness follows by the same arguments as for Bucklin, by considering the setting where every voter approves of all candidates. 
For the unweighted case, NP-completeness of BV-CUSB follows immediately from the fact that the possible winner problem for Bucklin is NP-complete (see the papers of Konczak and Lang [KL05], for the definition of the possible winner problem, and of Xia and Conitzer [XC11], for the result regarding Bucklin) and the fact that, for a given voting rule, the possible winner problem reduces to the swap bribery problem [EFS09]. However, on the one hand, the hardness of the possible winner problem was established for the simplified variant of Bucklin's rule only, and on the other hand, we can show that BV-CUSB is NP-complete even for elections with just two voters.

\section{Theorem 5.4 BV-CUSB is NP-complete, even for elections with two voters.}

Proof. It is easy to see that BV-CUSB is in NP. We show NP-hardness by a reduction from the following problem (which we will refer to as Single-Vote SWAP BRIBERY): Given a vote $v$ (expressed as a preference order over some candidate set $C$ ), a swap-bribery price function $\pi$ for $v$, a designated candidate $p \in C$, and two nonnegative integers $\ell$ and $k$, is there a sequence of swaps of adjacent candidates, of total cost at most $k$, that ensures that $p$ is ranked among the top $\ell$ positions in $v$. (Elkind et al. [EFS09] studied this problem as a variant of the swap bribery problem for $k$-approval elections, where $k$ is part of the input and the election consists of a single vote; they established NP-completeness of the problem in their Theorem 6.)

Let $I=(C, v, \pi, p, \ell, k)$ be an instance of Single-Vote SwAP BRIBERY. We form a Bucklin election $E=(A, V)$ as follows. Let $C^{\prime}$ be a collection of some $\|C\|-1$ dummy candidates. We set $A=C \cup C^{\prime} \cup\{d\}$. We partition $C^{\prime}$ into two sets, $C_{1}^{\prime}$ and $C_{2}^{\prime}$, such that $\left\|C_{1}^{\prime}\right\|=\ell$ and $\left\|C_{2}^{\prime}\right\|=\left\|C^{\prime}\right\|-\ell$. (We pick any easily computable partition.) We let $V$ be a collection of two voters, $v_{1}$ and $v_{2}$, with price functions $\pi_{1}$ and $\pi_{2}$ :

1. $v_{1}$ has preference order $d>v>C^{\prime}$ (i.e., $v_{1}$ ranks $d$ on the top position, then all the candidates from $C$ in the same order as $v$, and then all the candidates from $C^{\prime}$, in some arbitrary-buteasy-to-compute order). For each two candidates $x, y \in A$, if both $x$ and $y$ are in $C$ then we set $\pi_{1}(x, y)=\pi(x, y)$, and otherwise we set $\pi_{1}(x, y)=k+1$.

2. $v_{2}$ has preference order $p>C_{1}^{\prime}>d>C_{2}^{\prime}>C-\{p\}$ (that is, $v_{2}$ ranks $p$ first, then $\ell$ candidates from $C_{1}^{\prime}$ followed by $d$, followed by the remaining candidates from $C^{\prime}$, and, then, followed by the candidates from $C-\{p\})$. For each two candidates $x, y \in A$, we set $\pi_{2}(x, y)=k+1$.

Note that in our election $\operatorname{maj}(V)=2$ and, if $p$ is not among the top $\ell$ positions within $v, d$ is a winner with Bucklin score $\ell+2$ (we cannot say that $d$ is the unique winner because we do not know on what position $p$ is ranked in $v$ ). We claim that $p$ can become a unique Bucklin winner of election $E$ through a swap bribery of cost at most $k$ if and only if $I$ is a yes-instance of SINGLE-VOTE SwAP BRIBERY.

Assume that $I$ is a yes-instance of SINGLE-VOTE SWAP BRIBERY. This means that there is a sequence of swaps within $v$ after which $p$ is ranked among the top $\ell$ positions in $v$. Applying the same swaps to $v_{1}$ would cost the same and would put $p$ among top $\ell+1$ positions in $v_{1}$, making $p$ the unique Bucklin winner.

On the other hand, assume that there is a cost-at-most- $k$ sequence of swaps within $V$ that make $p$ a unique Bucklin winner. Since any swap that is not in the $v$ part of $v_{1} \operatorname{costs} k+1$, we have that $d$ 's Bucklin score is still $\ell+2$, and, thus, after the swaps, $p$ 's Bucklin score is in $\{2, \ldots, \ell+$ 
1\}. Executing the same swaps within $v$ shows that $I$ is a yes-instance of Single-Vote SwaP BRIBERY.

To establish that BV-DUSB also is NP-complete for the case of two voters, it suffices to use the same construction as above, with the exception that now (a) $d$ is the designated candidate whose victory we want to preclude, and (b) $v_{2}$ ranks $d$ on position $\ell+1$ (and not $\ell+2$ ). Analogous results for the fallback rule follow immediately.

Corollary 5.5 BV-DUSB, FV-CUSB, and FV-DUSB are NP-complete even for the cases of two voters.

\subsection{Results for Extension Bribery}

Let us now move on to the study of extension bribery. The following observation will simplify our discussion.

Observation 5.6 In (constructive) extension bribery problems for the fallback rule it is never profitable to extend any vote in any other way than by asking the voter to include the designated candidate on the last unranked position.

Thus we will often specify the extension bribery price functions by simply giving the cost of extending the vote by just one candidate (we will refer to this number as extension cost of the vote).

Not surprisingly, the weighted variants of extension bribery are NP-complete.

\section{Theorem 5.7 Both FV-CWEB and FV-DWEB are NP-complete.}

Proof. Obviously, FV-CWEB is in NP. To show NP-hardness, we use a reduction from PARTITION. Let $\left(\{1, \ldots, k\},\left(a_{1}, \ldots, a_{k}\right)\right)$ be an instance of PARTITION. We define a fallback election $(C, V)$ with the candidate set $C=\{b, c, p\}$, the designated candidate $p$, and $V$ consisting of the following $k+2$ voters:

1. There is one voter $v_{0}$ with the ballot $p \mid\{b, c\}$, with weight $K+1$ and extension cost $K+1$.

2. For each $i, 1 \leq i \leq k$, there is a voter $v_{i}$ who casts the ballot $c \mid\{b, p\}$, has weight $w_{i}=a_{i}$, and has extension cost $a_{i}$.

3. There is one voter $v_{k+1}$ who casts the ballot $b \mid\{c, p\}$ with weight $2 K$ and extension cost $K+1$.

The total sum of the voter's weights in this election is $5 K+1$, so $\operatorname{maj}(V)>2 K$. The weighted scores of the candidates in $(C, V)$ are shown in Table 9(a). Both $c$ and $b$ are fallback winners in $(C, V)$ and they win by approval, thus $p$ is not a (unique) fallback winner in $(C, V)$.

We claim that there is a set $A^{\prime} \subseteq A=\{1, \ldots, k\}$ such that $\sum_{i \in A^{\prime}} a_{i}=\sum_{i \notin A^{\prime}} a_{i}=K$ if and only if $p$ can be made the unique fallback winner by extension-bribing some of the voters without exceeding the budget $K$.

From left to right: Suppose that there is a set $A^{\prime} \subseteq A$ such that $\sum_{i \in A^{\prime}} a_{i}=\sum_{i \notin A^{\prime}} a_{i}=K$. Change the votes of those voters $v_{i}$ with $i \in A^{\prime}$ from $c \mid\{b, p\}$ to $c>p \mid\{b\}$. Each of these changes costs $a_{i}$, 
Table 9: Scores in the election constructed in the proof of Theorem $\underline{5.7}$

\begin{tabular}{|c|c|c|c|c|c|c|c|}
\hline & $b$ & $c$ & $p$ & & $b$ & $c$ & $p$ \\
\hline score $^{1}$ & $\overline{2 K}$ & $2 K$ & $\overline{K+1}$ & score $^{1}$ & $2 K$ & $2 K$ & $K+1$ \\
\hline & & & & score $^{2}$ & $2 K$ & $2 K$ & $2 K+1$ \\
\hline
\end{tabular}

so the total cost is $K$. The candidates' scores in the resulting election $\left(C, V^{\prime}\right)$ are shown in Table $\underline{9(\mathrm{~b})}$. We see that $p$ is the unique fallback winner in the bribed election.

From right to left: Suppose that $p$ is the unique fallback winner in the election $\left(C, V^{\prime}\right)$, where $V^{\prime}$ is the changed voter set and the corresponding changes cost at most $K$. Hence, the only changes that can be made (and that follow Observation 5.6) are adding the candidate $p$ to the approval strategies of some of the voters $v_{1}, \ldots, v_{k}$. The scores of the candidates $b$ and $c$ cannot be decreased, so $p$ has to gain $K$ points to have strictly more points than $b$ and $c$. Thus, there exists a set $A^{\prime} \subseteq A$ such that $\sum_{i \in A^{\prime}} a_{i}=\sum_{i \notin A^{\prime}} a_{i}=K$ and $p$ has to be added to the approval strategies of those voters $v_{i}$ with $i \in A^{\prime}$.

The destructive case can be proven by changing the role of candidates $p$ and $c$ and changing the weights of both $v_{0}$ and $v_{k+1}$ to $K$.

On the other hand, the unweighted variant of the problem is in P. This is a nice complement to the hardness results of Schlotter et al. [SFE11] regarding support bribery. The main difference regarding support bribery and extension bribery is that under the former we assume the voters to rank all the candidates but declare as approved only some of their top candidates, whereas in the latter (and, in general, in our model) we assume the voters to rank only the approved candidates and completely disregard the disapproved candidates.

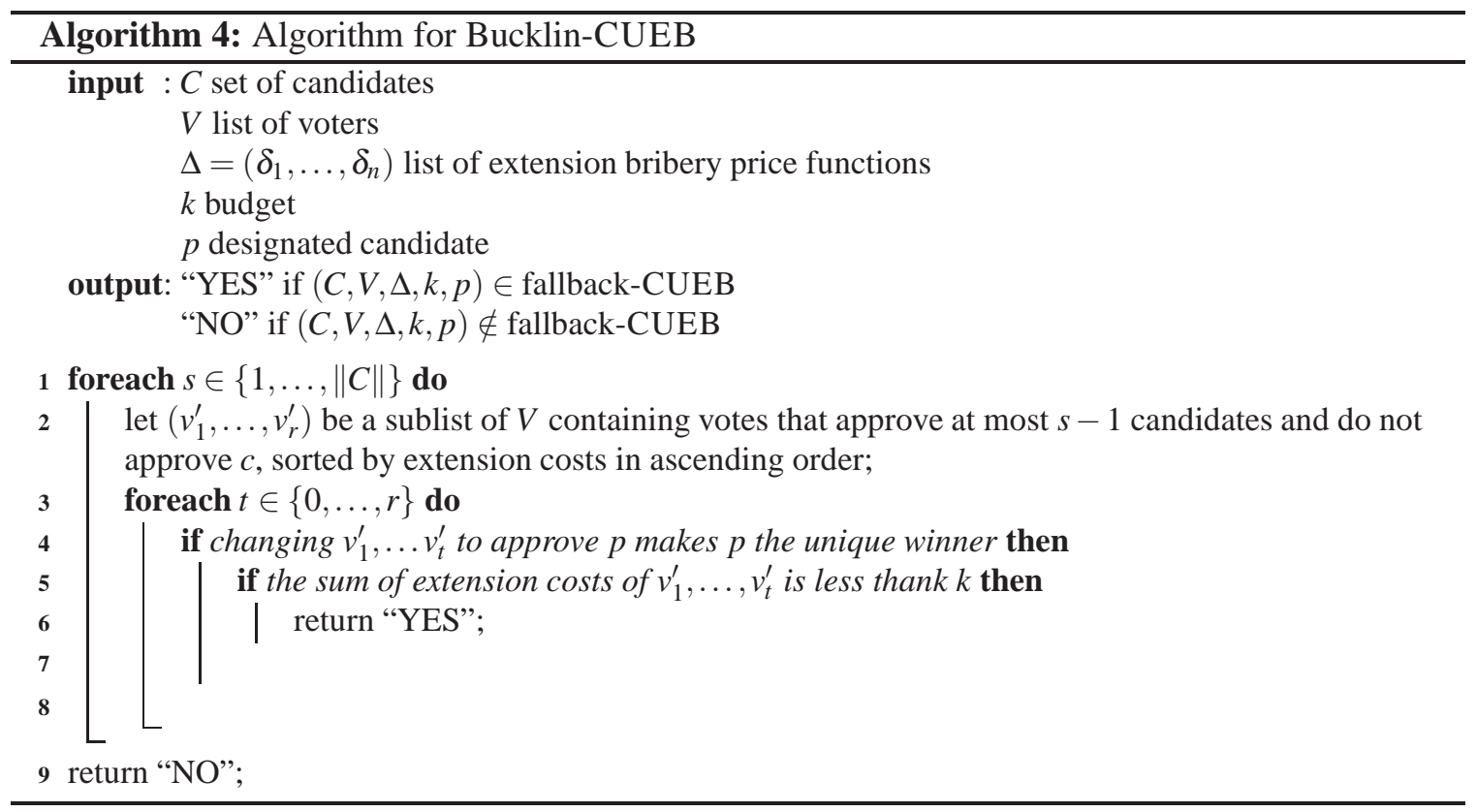


Theorem 5.8 FV-CUEB and FV-DUEB are in $\mathrm{P}$.

Proof. Let us consider FV-CUEB first. We claim that Algorithm $\underline{4}$ solves the problem in polynomial time. The algorithm considers each round $s$ in which $p$ could possibly become the unique winner and tries the cheapest bribery that might achieve it. The algorithm clearly runs in polynomial time and its correctness follows by Observation 5.6.

It is clear how to adapt Algorithm $\underline{4}$ to the case of nonunique winners. Then, to solve the destructive variant of the problem it suffices to check if any candidate other than $p$ can be made a nonunique winner within the budget.

\section{References}

[BCE13] F. Brandt, V. Conitzer, and U. Endriss. Computational social choice. In G. Weiß, editor, Multiagent Systems, pages 213-283. MIT Press, second edition, 2013.

$\left[\mathrm{BEH}^{+} 10\right]$ D. Baumeister, G. Erdélyi, E. Hemaspaandra, L. Hemaspaandra, and J. Rothe. Computational aspects of approval voting. In J. Laslier and R. Sanver, editors, Handbook on Approval Voting, chapter 10, pages 199-251. Springer, 2010.

[BF78] S. Brams and P. Fishburn. Approval voting. American Political Science Review, 72(3):831-847, 1978.

[BF83] S. Brams and P. Fishburn. Approval Voting. Birkhäuser, Boston, 1983.

[BFLR12] D. Baumeister, P. Faliszewski, J. Lang, and J. Rothe. Campaigns for lazy voters: Truncated ballots. In Proceedings of the 11th International Joint Conference on Autonomous Agents and Multiagent Systems, pages 577-584. IFAAMAS, June 2012.

[BO91] J. Bartholdi III and J. Orlin. Single transferable vote resists strategic voting. Social Choice and Welfare, 8(4):341-354, 1991.

[BS06] S. Brams and R. Sanver. Critical strategies under approval voting: Who gets ruled in and ruled out. Electoral Studies, 25(2):287-305, 2006.

[BS09] S. Brams and R. Sanver. Voting systems that combine approval and preference. In S. Brams, W. Gehrlein, and F. Roberts, editors, The Mathematics of Preference, Choice, and Order: Essays in Honor of Peter C. Fishburn, pages 215-237. Springer, 2009.

[BTT89] J. Bartholdi III, C. Tovey, and M. Trick. The computational difficulty of manipulating an election. Social Choice and Welfare, 6(3):227-241, 1989.

[BTT92] J. Bartholdi III, C. Tovey, and M. Trick. How hard is it to control an election? Mathematical and Computer Modelling, 16(8/9):27-40, 1992.

[CSL07] V. Conitzer, T. Sandholm, and J. Lang. When are elections with few candidates hard to manipulate? Journal of the ACM, 54(3):Article 14, 2007. 
[DS12] B. Dorn and I. Schlotter. Multivariate complexity analysis of swap bribery. Algorithmica, 64(1):126-151, 2012.

[EF10a] E. Elkind and P. Faliszewski. Approximation algorithms for campaign management. In Proceedings of the 6th International Workshop On Internet And Network Economics, pages 473-482. Springer-Verlag Lecture Notes in Computer Science \#6484, December 2010.

[EF10b] G. Erdélyi and M. Fellows. Parameterized control complexity in Bucklin voting and in fallback voting. In V. Conitzer and J. Rothe, editors, Proceedings of the 3rd International Workshop on Computational Social Choice, pages 163-174. Universität Düsseldorf, September 2010.

[EFRS12] G. Erdélyi, M. Fellows, J. Rothe, and L. Schend. Control complexity in Bucklin and fallback voting. Technical Report arXiv:1103.2230 [cs.CC], Computing Research Repository, arXiv.org/corr/, March 2012. March, 2011. Revised August, 2012. Extends the AAMAS-2011 paper [EPR11].

[EFS09] E. Elkind, P. Faliszewski, and A. Slinko. Swap bribery. In Proceedings of the 2nd International Symposium on Algorithmic Game Theory, pages 299-310. Springer-Verlag Lecture Notes in Computer Science \#5814, October 2009.

[ENR09] G. Erdélyi, M. Nowak, and J. Rothe. Sincere-strategy preference-based approval voting fully resists constructive control and broadly resists destructive control. Mathematical Logic Quarterly, 55(4):425-443, 2009.

[EPR11] G. Erdélyi, L. Piras, and J. Rothe. The complexity of voter partition in Bucklin and fallback voting: Solving three open problems. In Proceedings of the 10th International Joint Conference on Autonomous Agents and Multiagent Systems, pages 837-844. IFAAMAS, May 2011.

[ER10] G. Erdélyi and J. Rothe. Control complexity in fallback voting. In Proceedings of Computing: the 16th Australasian Theory Symposium, pages 39-48. Australian Computer Society Conferences in Research and Practice in Information Technology Series, vol. 32, no. 8, January 2010.

[FHH09] P. Faliszewski, E. Hemaspaandra, and L. Hemaspaandra. How hard is bribery in elections? Journal of Artificial Intelligence Research, 35:485-532, 2009.

[FHHR09] P. Faliszewski, E. Hemaspaandra, L. Hemaspaandra, and J. Rothe. Llull and Copeland voting computationally resist bribery and constructive control. Journal of Artificial Intelligence Research, 35:275-341, 2009.

[FP10] P. Faliszewski and A. Procaccia. AI's war on manipulation: Are we winning? AI Magazine, 31(4):53-64, 2010.

[GJ79] M. Garey and D. Johnson. Computers and Intractability: A Guide to the Theory of NP-Completeness. W. H. Freeman and Company, 1979. 
[HHR07] E. Hemaspaandra, L. Hemaspaandra, and J. Rothe. Anyone but him: The complexity of precluding an alternative. Artificial Intelligence, 171(5-6):255-285, 2007.

[KL05] K. Konczak and J. Lang. Voting procedures with incomplete preferences. In Proceedings of the Multidisciplinary IJCAI-05 Workshop on Advances in Preference Handling, pages 124-129, July/August 2005.

[Men11] C. Menton. Normalized range voting broadly resists control. Technical Report arXiv:1005.5698v2 [cs.GT], Computing Research Repository, arXiv.org/corr/, April 2011. Revised June, 2012. To appear in Theory of Computing Systems.

[Pap95] C. Papadimitriou. Computational Complexity. Addison-Wesley, second edition, 1995.

[Rot05] J. Rothe. Complexity Theory and Cryptology. An Introduction to Cryptocomplexity. EATCS Texts in Theoretical Computer Science. Springer-Verlag, 2005.

[RS] J. Rothe and L. Schend. Challenges to complexity shields that are supposed to protect elections against manipulation and control: A survey. Annals of Mathematics and Artificial Intelligence. To appear.

[RS12] J. Rothe and L. Schend. Control complexity in Bucklin, fallback, and plurality voting: An experimental approach. In Proceedings of the 11th International Symposium on Experimental Algorithms, pages 356-368. Springer-Verlag Lecture Notes in Computer Science \#7276, June 2012.

[SFE11] I. Schlotter, P. Faliszewski, and E. Elkind. Campaign management under approvaldriven voting rules. In Proceedings of the 25th AAAI Conference on Artificial Intelligence, pages 726-731, August 2011.

[XC11] L. Xia and V. Conitzer. Determining possible and necessary winners given partial orders. Journal of Artificial Intelligence Research, 41:25-67, 2011.

[Xia12] L. Xia. Computing the margin of victory for various voting rules. In Proceedings of the 13th ACM Conference on Electronic Commerce, pages 982-999. ACM Press, 2012.

[XZP ${ }^{+}$09] L. Xia, M. Zuckerman, A. Procaccia, V. Conitzer, and J. Rosenschein. Complexity of unweighted coalitional manipulation under some common voting rules. In Proceedings of the 21st International Joint Conference on Artificial Intelligence, pages 348-353. IJCAI, July 2009. 